\title{
The Globular Cluster Systems in the Coma Ellipticals. IV: WFPC2 Photometry for Five Giant Ellipticals ${ }^{12}$
}

\author{
William E. Harris \\ Department of Physics and Astronomy, McMaster University, Hamilton ON L8S 4M1, Canada \\ harris@physics.mcmaster.ca \\ J. J. Kavelaars \\ Herzberg Institute of Astrophysics, National Research Council, 5017 West Saanich Road, Victoria \\ BC V9E $2 E^{r}$ \\ jj.kavelaars@nrc-cnrc.gc.ca \\ David A. Hanes \\ Department of Physics, Queen's University, Kingston ON KYL 3N6, Canada \\ hanes@astro.queensu.ca \\ Christopher J. Pritchet \\ Department of Physics and Astronomy, University of Victoria, Box 3055, Victoria, BC V8W \\ 3P6, Canada \\ pritchet@phys.uvic.ca \\ W. A. Baum \\ Department of Astronomy, University of Washington, Box 351580, Seattle WA 98195 \\ baum@astro.washington.edu
}

\begin{abstract}
We analyze photometric data in $V$ and $I$ for the globular cluster (GC) systems in five of the giant ellipticals in the Coma Cluster: NGC 4874, 4881, 4889, 4926, and IC 4051. All of the raw data, from the Hubble Space Telescope WFPC2 Archive, are analyzed in a homogeneous way so that their five cluster systems can be strictly intercompared. We find that the GC luminosity functions are quite similar to one another and reinforce the common nature of the mass distribution of old, massive star clusters in gE galaxies. The GCLF turnover derived from a composite sample of more than 9, 000 GCs appears at $V=27.71 \pm 0.07\left(M_{V}=-7.3\right)$, and our data reach about half a magnitude fainter
\end{abstract}


than the turnover. We find that both a simple Gaussian curve and an evolved Schechter function fit the bright half of the GCLF equally well, though the Coma GCLF is broader and has a higher "cutoff mass" $\left(M_{c} \sim 3 \times 10^{6} M_{\odot}\right)$ than in any of the Virgo giants. These five Coma members exhibit a huge range in GC specific frequency, from a low of $S_{N} \simeq$ 0.7 for NGC 4881 up to $\simeq 12$ for IC 4051 and NGC 4874. No single formation scenario appears able to account for these differences in otherwise-similar galaxies and may require carefully prescribed differences in their merger history, gas-free versus gas-rich progenitors, GC formation efficiency, initial density of environment, or tidal harassment within the Coma potential well. The supergiant cD galaxy NGC 4874 has the richest globular cluster system known, probably holding more than 30,000 clusters; its true extent is not yet determined and may extend well out into the Coma potential well. For the three biggest GC systems (NGC 4874, 4889, IC 4051), analysis of the ( $V-I)$ color distributions shows that all three populations are dominated by red, metal-rich clusters. Their metallicity distributions also may all have the normal bimodal form, with the two sequences at mean colors $\langle V-I\rangle$ (blue $) \simeq 0.98$ and $\langle V-I\rangle$ (red) $\simeq 1.15$. These values fall along the previously established correlations of mean color with galaxy luminosity. However, the color distributions and relative numbers of metal-rich clusters show intriguing counterexamples to a trend established by Peng et al. 2008 (ApJ 681, 197) for the Virgo galaxies. For the brightest Virgo ellipticals, they find that the red GCs make up only $\sim 30 \%$ of the cluster population, whereas in our similarly luminous Coma galaxies they make up more than half. At the very highest-density and most massive regimes represented by the Coma supergiants, formation of metal-rich clusters seems to have been especially favored.

Subject headings: Galaxies: Formation - Galaxies: Individual - Galaxies: Star Clusters

\section{Introduction}

The Coma cluster is the nearest example of an Abell cluster which holds a truly rich collection of E/S0 galaxies in a dynamically evolved environment. As such, it provides a unique locale for the study of globular cluster systems (GCSs) under higher-density and dynamically "older" environmental conditions than in Virgo, Fornax, and smaller nearby groups of galaxies Harris 2001).

\footnotetext{
${ }^{1}$ Based on observations with the NASA/ESA Hubble Space Telescope, obtained at the Space Telescope Science Institute, which is operated by the Association of Universities for Research in Astronomy, Inc., under NASA contract NAS 5-26555.

${ }^{2}$ This research used the facilities of the Canadian Astronomy Data Centre operated by the National Research Council of Canada with the support of the Canadian Space Agency.
} 
The first HST-based photometry of a GCS in Coma was for NGC 4881 by Baum et al. (1995), a giant elliptical in the cluster core. In Papers I and II of our series on the Coma cluster (Kavelaars et al. 2000; Harris et al. 2000), we discussed the GCS in NGC 4874, the supergiant cD-type elliptical that lies near the center of the larger Coma potential well. In Baum et al. (1997) and in our Paper III (Woodworth \& Harris 2000), similar material was presented for IC 4051, another giant on the outskirts of the Coma cluster core region. In the present paper, we discuss new results (GCS radial distribution, total population, metallicity distribution, and luminosity function) for two additional Coma members, NGC 4889 and 4926, that have not previously been published. Finally, we reanalyze the WFPC2 Archive data for NGC 4874, 4881, and IC4051 to ensure that all of it is on a homogeneous photometric basis, and discuss all five galaxies together.

The three Coma members that we studied earlier through HST (NGC 4874, 4881, IC 4051) already present a striking range of GCS properties. NGC 4881 has an extremely low-specificfrequency system that is quite surprising for a giant $\mathrm{E}$ galaxy in such a rich environment. By contrast, IC 4051 has a system in the high- $S_{N}$ range that would normally be associated with central supergiant cD galaxies (Harris 2001), but one that is also spatially quite compact, perhaps due to severe tidal truncation from the Coma potential. NGC 4874, the central supergiant, has a high- $S_{N}$ system with a very large radial extent, perhaps spanning the entire Coma core. These systems hint that we may not yet have seen the entire variety of GCS characteristics that this environment has to show.

The globular cluster populations in the Coma galaxies have also attracted attention through ground-based imaging, although their $\sim 100-\mathrm{Mpc}$ distance has always made such efforts extremely challenging. Harris (1987) and Thomsen \& Baum (1987) first obtained deep enough imaging to reveal the GCs in NGC 4874 with cameras on the CFHT, though these studies were useful for no more than the roughest estimates of specific frequency (that is, the number of GCs per unit galaxy luminosity). Blakeslee \& Tonry (1995) used a combination of surface brightness fluctuation (SBF) techniques and resolved-GC photometry to estimate the GC populations in NGC 4874 and 4889. More recently, Marin-Franch \& Aparicio (2002) used SBF to measure the cluster populations in 17 Coma galaxies. From this survey, they confirmed the rather bewildering variety of specific frequencies from galaxy to galaxy - from a cD-like high of $S_{N} \simeq 12$ down to the $S_{N} \sim 1$ level that we normally associate with much more GC-poor spiral and dwarf galaxies. Furthermore, from their entire sample of 17 galaxies there is no trend of $S_{N}$ with either galaxy luminosity or location (radius from Coma center). However, by restricting their sample to only their five galaxies within "subgroup 1" of Gurzadyan \& Mazure (2001), which is the subcluster centered on NGC 4874 itself, they conclude that $S_{N}$ may indeed be correlated with environment in the sense that $S_{N}$ decreases with increasing distance from the group center. A similar trend had already been proposed by Blakeslee et al. (1997) from a sample of specific frequencies for 23 galaxies in 19 rich clusters. This trend is also reminiscent of the new discussion by Peng et al. (2008) for the Virgo galaxies, in which they show that the $\mathrm{dE}$ galaxies in Virgo have higher $S_{N}$ values closer to the central giant M87.

Although SBF photometry has proven to be effective in measuring the total GC populations 
in E galaxies, it cannot say much about other essential characteristics such as the GC color or luminosity functions, which are the key visible tracers of the GC metallicity and mass distributions. For these, we need the deep photometry of individual GCs that the HST cameras can provide. In Coma, there are five such galaxies with available WFPC2 imaging data that we can discuss within a strictly homogeneous photometric system. These are summarized in Table 1, where we list the galaxy luminosity (with total magnitudes adopted from RC3), heliocentric radial velocity, and projected distance from the center of Coma (assumed to be at NGC 4874). For comparison, the core radius of the entire Coma galaxy distribution is $r_{c} \simeq 15^{\prime}-20^{\prime}$ (see Paper II) and its overall mean heliocentric velocity is $6917 \mathrm{~km} \mathrm{~s}^{-1}$ (Colless \& Dunn 1996).

Much previous work in the literature can be found on the spatial and velocity substructures within Coma. To first order, identifiable subclusters can be found centered on the supergiant $\mathrm{cD}$ galaxy NGC 4874 (the center of the biggest subsystem), the other outlying cD NGC 4839, and the supergiant NGC 4889 (for much more detailed discussions, see Fitchett \& Webster 1987; Baier et al. 1990; Merritt \& Tremblay 1994; Colless \& Dunn 1996; Gurzadyan \& Mazure 2001, among others). These subgroupings also appear in the intracluster X-ray gas (Neumann et al. 2003; Adami et al. 2005). NGC 4889 has a total luminosity comparable with NGC 4874, but is structurally more compact than NGC 4874, without a cD-type envelope. The other three systems in our WFPC2 study (NGC 4881, 4926, IC 4051) are more normal giant ellipticals and are all among the ten brightest Coma members. Only NGC 4926 is clearly outside the main cluster core.

In the following sections, we describe the raw data used for our program, the subsequent analysis, and lastly a comparison of the five galaxies. We assume here a distance modulus for Coma of $(m-M)_{0}=34.97 \pm 0.13$ or $d=98.6 \pm 6.1 \mathrm{Mpc}$. This distance results from our adopted redshift of $7100 \pm 200 \mathrm{~km} \mathrm{~s}^{-1}$ (Colless \& Dunn 1996; Kavelaars et al. 2000) and a Hubble constant

$h=0.72 \pm 0.04$ (Freedman et al. 2001; Spergel et al. 2007). At this distance, 10 arcseconds on the sky is equivalent to a linear scale of $4.8 \mathrm{kpc}$.

\section{The Database}

The raw WFPC2 data for these galaxies were all long exposures drawn from the HST Archive. The images were originally acquired as parts of programs GO-5233, 6283 (NGC 4881 and IC 4051; Westphal PI) and GO-6104, 8200 (NGC 4874, 4889, 4926; Harris PI). Total exposure times and filters are summarized in Table 2, The raw capabilities of the WFPC2 camera are now exceeded by the newer ACS and WFC3, which have wider fields of view and higher sensitivity particularly in the blue. Eventually, it will be possible (for example) to study the color/metallicity distributions and spatial distributions of the Coma galaxy GCs with higher precision with these better cameras (Carter et al. 2008). However, the WFPC2 images for four of these Coma targets already reach quite far into their globular cluster luminosity functions (GCLFs) and, to this point, make up the deepest available sample of Coma GC material to work with. For this reason, we believe it is worth discussing the combined material in a homogeneous way. We will concentrate on two main 
questions: first, are their GCLFs the "standard" Gaussian-like ones seen in other large E galaxies? And second, how similar are their color distributions to one another and to the conventional bimodal form found in other large galaxies, as far as we can gauge them within the limitations of the $(V-I)$ color index?

Our data reduction follows the same procedures outlined in detail in Papers I-III. Master $V$ and $I$ frames free of cosmic rays and bad-pixel artifacts were generated by registering and median-combining the individual WFPC2 exposures. When combining the exposure sequences, we treated each of the four CCD chips separately (we did not rely on the Archive WFPC2 Associations, performing the image combining operations ourselves from the raw exposures). For all five fields, the PC1 chip is centered on the galaxy. To facilitate the detection of faint starlike objects (which include the globular clusters we are looking for; see below), on the combined PC1 images we generated a smooth model of the galaxy isophotes with the STSDAS/ELLIPSE package and subtracted it from the image. In the WF2,3,4 frames we median-filtered the image to remove the more gradual isophotal gradient from the outer parts of the galaxy. These steps were done iteratively along with the DAOPHOT star-finding and removal to produce the cleanest possible master images for final stellar photometry.

At the 100-Mpc distance of Coma, a typical globular cluster half-light diameter of $\simeq 6 \mathrm{pc}$ subtends $0.012^{\prime \prime}$, an order of magnitude smaller than the resolution of the telescope. This feature of the data makes the GCs indistinguishable from stars in appearance, and carries two major advantages for the photometry: first, it is straightforward to carry out normal PSF-style object detection and photometry on these images; and second, most of the background population of small, faint galaxies can be easily distinguished and removed from the sample, minimizing the field contamination.

In Figure 1 we show the results of some simulations that demonstrate the validity of the PSFfitting procedure for this material. Here, we have used the tools in the baolab/ishape code of Larsen (1999) to generate simulated cluster profiles of various half-light radii $r_{h}$ in a realistic range; project them to the Coma distance; convolve them with the WFPC2 point spread function; and finally place them into an image for remeasurement. For these tests we specifically used our empirical point spread function derived for the NGC 4926 WF fields, but any of the other targets would have done equally well. As is extensively discussed by Larsen (1999), a PSF-convolved cluster profile will yield a measurable and systematically correct $r_{h}$ with the use of fitting codes like ishape if the $F W H M$ of the intrinsic cluster profile is larger than about 10 percent of the $F W H M$ of the PSF itself. Objects smaller than this are effectively indistinguishable from stars.

Fig. 11 shows an equivalent way to express this result. We generate PSF-convolved profiles for model clusters of different radii: $r_{h}=0,3,6,10,20$ parsecs. The smallest $r_{h}$ obviously corresponds to a pure stellar profile; $r_{h}=3 \mathrm{pc}$ corresponds to an average Milky Way globular cluster (see the data in Harris 1996); $r_{h}=6 \mathrm{pc}$ is at the upper end of the normal GC distribution; and $r_{h}=10$ and $20 \mathrm{pc}$ correspond to profiles for more extended objects resembling $\mathrm{dE}$ nuclei or Ultra-Compact 
Dwarfs (UCDs); see, for example, Evstigneeva et al. (2008). For the template GC profiles we used the KING30 model in ishape, which closely matches the mean central concentration of real GCs (Harris 1996; Larsen 1999). In Fig. 1, the curves of growth (aperture magnitude enclosed within radius $r$ in pixels) are plotted for each of the five models. At the measurement radius of $2 \mathrm{px}$ that we adopted for all our photometry, we then read off the magnitude difference $\Delta m$ between the pure stellar curve $\left(r_{h}=0\right)$ and the curve for the more extended object. This difference gives us the magnitude correction that we would have to apply to the actual, PSF-convolved GC profile in order to put its measured magnitude onto the same scale as a pure PSF. In principle, we could then use ishape to solve for all the individual $r_{h}$ values and then use this grid of curves to read off the necessary magnitude correction to apply to each one.

In practice, what we find is that the curve for $r_{h}=3 \mathrm{pc}$ (that is, a "baseline normal" GC) is indistinguishable from that of a star. For any $r_{h}<6 \mathrm{pc}$, the correction is $\Delta m<0.01$ mag and thus negligible compared with the internal scatter of the photometry (see below). It is only for UCD-like systems at $r_{h} \sim 20 \mathrm{pc}$ where the correction approaches $\sim 0.1$ mag. As further confirmation, we note the work of Wehner \& Harris (2007) for the NGC 3311 globular cluster system, which is at roughly half the Coma distance. Even there, only a handful of the largest, brightest, UCD-like clusters could be seen to be marginally resolved on WFPC2 images.

The object-finding and photometry was carried out with the normal DAOPHOT/ALLSTAR codes (Stetson 1994). In each field, in each of the $V$ and $I$ filters, and on each one of the four camera quadrants (PC1, WF2,3,4 independently), we generated empirical point spread functions (PSFs) from moderately bright isolated stars. The instrumental magnitudes returned by these codes were CTE-corrected, then transformed to the standard (V,I) system (Holtzman et al. 1995) after aperture correction to the nominal $0.5^{\prime \prime}$ radius specified by the transformations.

A problem encountered at this stage was that the fields of some of our galaxies (NGC 4926 and 4881 particularly) are so "clean" of foreground stars - more so than in the other Coma fields - that the PSFs, in the end, relied on fewer and fainter stars than we would have preferred, particularly in the $I$-band frames which had shorter total exposure and thus lower signal-to-noise than the $V$ frames. The main step which might be affected adversely is the aperture correction (mean difference between the PSF-fitted instrumental magnitudes and the 0.5 whole-aperture magnitudes) and thus the zeropoint of the final $I$ photometric scale. Although the $V$ magnitude scale should be correct to $\pm 0.02 \mathrm{mag}$, the scale in $I$ may still be uncertain chip-to-chip to no better than $\pm 0.05 \mathrm{mag}$, a worry which is borne out to some extent in the dispersion of the cluster colors once we combine the data from all four chips (see below).

A high proportion of the background contamination (mostly faint, slightly nonstellar background galaxies) can be eliminated objectively, first through the use of radial-moment image analysis and second through rejection of any objects not found in both filters (extreme red or blue colors). As in our previous papers, for the radial moments we use the $r_{1}$ moment as implemented in Harris et al. (1991) along with artificial-star tests to define the boundaries of the stellar sequence 
(see Papers I and III for complete discussion).

Lastly, artificial-star simulations of the same type used in our previous work were used to define the completeness of detection $f(V, I)$, an important issue particularly for determining the GC luminosity function. Even in the innermost regions around each galaxy, the photometry is not affected by crowding, so the completeness and internal measurement scatter are determined only by the faintness of each object and the surface brightness of its local background. Averaged results over many simulated runs (where we add several hundred artificial stars to the images, then re-reduce them through the same DAOPHOT measurement sequence) were used to define the $f$-distributions for each field and each chip. One sample of these is shown in Figure 2, Approximate fits to the trend of $f$ with magnitude are given by modified versions of the interpolation functions used in our previous papers,

$$
f=0.5\left(1-\frac{\alpha\left(m-m_{0}\right)}{\sqrt{1+\alpha^{2}\left(m-m_{0}\right)^{2}}}\right)
$$

The two free parameters in this function are the "completeness limit" $m_{0}$ at which $f$ reaches $50 \%$; and the slope $\alpha$, which governs how steeply $f$ declines as it passes through the $m_{0}$ midpoint (see Fig. 2). The various values of $\left(m_{0}, \alpha\right)$ that we found from the artificial-star tests are given in Table 3. For the same field, $m_{0}$ is noticeably different on the PC1 chip than on the three WF chips in the sense that PC1 does not reach as faint. This difference is primarily due to the much higher background light on PC1. We found no dependence of the limiting magnitude on galactocentric radius for the $\mathrm{WF}$ chips. For the $\mathrm{PC} 1$ fields, $m_{0}$ becomes noticeably brighter within $\simeq 5^{\prime \prime}$ of the galaxy centers, but as will be seen below, we made little or no use of the data in this innermost region, and none of our conclusions depend significantly on it.

The artificial-star tests showed that the random measurement uncertainties are reasonably well described by a simple interpolation function $e(m) \simeq 0.03+a \cdot \exp (V-b)$, for constants $a, b$. For NGC 4874, 4889, and IC 4051 we find $a=0.04, b_{V}=26.2, b_{I}=25.3$ : for NGC $4926 a=0.01, b_{V}=$ $25.2, b_{I}=23.4$; and for NGC $4881 a=0.04, b_{V}=24.5, b_{I}=24.5$. These approximations also match well with the internal photometric uncertainties returned by the ALLSTAR solutions themselves, consistent with cases such as this where crowding is quite unimportant and the measurement uncertainties are dominated simply by the object faintness and the local background light.

The artificial-star tests and plots of the raw data versus radius showed that within $r \simeq 4^{\prime \prime}$ (40 pixels, or $2 \mathrm{kpc}$ in linear distance) of the galaxy centers the data become severely incomplete because of the very high background light. The data within this innermost region around each galaxy were simply eliminated from our discussion.

In Figure 3 we show the spatial pattern of the brighter "starlike" objects $(V<26$ and detected in both $V$ and $I$ ) around each of the five galaxies. As will be seen below, the magnitude range $V<26$ includes the brighter portion of the GC luminosity function and includes only a small proportion of field contamination, so these graphs give a useful visual impression of the size and spatial extent of the GC distributions. A striking variety of spatial structures is already evident. 
The raw color-magnitude distributions (CMDs) for the GCs are shown in Figure 4, The large differences in total GC populations show up here as well, but it is also evident from the CMDs that the color distributions in $(V-I)$ are rather similar. We will quantify these features in the following sections, as well as the luminosity functions.

\section{The Spatial Distributions}

Assessing the intrinsic spatial distributions of the globular cluster systems around these galaxies, as well as their luminosity functions and color distributions, first requires knowing the level of background contamination. For NGC 4874 and 4889, we can estimate only upper limits to the background level (i.e., the number of field contaminating objects per unit area) from those images alone, because the GCS probably extends well beyond the borders of the WFPC2 images. Fortunately, for IC 4051 and NGC 4926 the story is different. These two GC systems are compact enough that the measured numbers of objects drop off to near-constant levels for radii beyond $\simeq 50^{\prime \prime}$ (equivalent to $25 \mathrm{kpc}$ ). Since the exposure depths for these four fields were very similar, and they are all located in the high-latitude Coma region, we therefore decided to use the outer parts of the NGC 4926 and IC 4051 fields to define an average "global" background distribution in magnitude and color, which could then be used for the three galaxies that had by far the most populous GCSs (namely, NGC 4874, 4889, and IC 4051 itself). The raw number-density plots for all the individual galaxies are shown in Figure 5. This plot shows the radial falloff of the GC population for the magnitude range $22.0<I<25.5$ over which the photometry is essentially $100 \%$ complete. Experiments with the IC 4051 and NGC 4926 fields led us to adopt the "global background field" as consisting of all measured objects farther than $80^{\prime \prime}$ from the center of IC 4051 and farther than $70^{\prime \prime}$ from the center of NGC 4926. The total area of this composite background region is $3.98 \operatorname{arcmin}^{2}$.

For NGC 4881 and 4926, which have GCSs that are both compact and sparsely populated, we used the outer regions of only their own fields to define a local background, preferring in this case not to average in data from other fields that might have been subtly different in measurement selection and intrinsic background population (any such minor differences are far less important for the enormously more populous GCSs in the other three). The limiting magnitudes of the NGC

4881 exposures are also shallower than for the other four and so both the GC numbers and the background are especially low for it.

The spatial distributions, after subtraction of background, are shown in Figure 6. Unless otherwise noted below, the GC counts are for $V<27$, well within the range of high completeness. Here, the distributions are shown in the classic E-galaxy form of $\log \sigma$ versus $r^{1 / 4}$. In each graph we superimpose the available surface-brightness photometry for each galaxy, in order to compare the GCS spatial structure with that of the integrated halo light of the parent galaxy. Individual comments are summarized below.

NGC 4874: The integrated light profiles are from Jorgensen et al. (1992) in $r$ (dashed line), and 
Peletier et al. (1990) in $R$ (solid line). At radii $r \gtrsim 5^{\prime}$, the surface brightness profile begins to overlap that of NGC 4889 and for both galaxies becomes much more difficult to trace further outward. Its GC system is clearly very extended, going well beyond the WFPC2 field boundaries (Paper II and Fig. 3). Despite these restrictions, it is already obvious that the GCS has a distinctly shallower profile than the halo isophotes. More detailed discussion of the radial profile can be found in Harris et al. (2000) and our conclusions here are the same. The primary difference in the current data is that we were able to use a global background field with strictly homogeneous photometry and twice the effective area. As we discussed in Paper II, an important point of emphasis peculiar to NGC 4874 is the long-standing question whether cD-type centrally dominant galaxies may have accreted a high fraction of their GCs from their intracluster environment. Notably, its GCS is quite a bit more centrally concentrated than is the Coma X-ray gas, which in the region close to NGC 4874 has a near-constant surface brightness (see Fig. 3 of Paper II for the specific comparison). If there is a GC population component with a similarly near-flat spatial distribution in the Coma core region, it clearly does not show up in any important way within $\sim 100 \mathrm{kpc}$ of the galaxy. For these reasons it is possible to argue that the GCS predominantly belongs to the central galaxy rather than to the Coma potential well at large.

NGC 4889: The surface brightness profiles are from Jorgensen et al. (1992) (dashed line) and Peletier et al. (1990) (solid line). For this galaxy, the results fall into a familiar pattern whereby the GCS is moderately more extended (shallower) than the halo light throughout the entire run of our data, though both have steeper profiles than their counterparts in NGC 4874. Combined with the moderate specific frequency and high metallicity distribution that we find for NGC 4889 (see below), we interpret it as a normal, though extremely high luminosity, elliptical.

For both NGC 4889 and 4874, much wider-field imaging with similar depth will be needed to gauge properly how extended their cluster systems are at larger radius, since for $r \gtrsim 5^{\prime}$ they overlap each other rather heavily. The hints from the current data are that the NGC 4874 system probably dominates more and more at larger radii because of its shallower profile, but this remains to be seen. Wider-field data are also the only way to assess the true contribution, if any, of intracluster GCs within the Coma core. A roughly similar case has been studied in the Hydra cluster (Wehner et al. 2008), where two giants NGC 3309 and 3311 sit close to each other in the cluster core and one of them (NGC 3311) is a central cD. With the right data, a numerical solution can be performed to derive the two total GC populations and spatial distributions simultaneously. For Hydra, the result shows that the cD NGC 3311 is dominant and NGC 3309 unimportant; but in Coma, both the central giants have very large cluster populations and the actual degree of overlap is not yet known.

IC 4051: The surface brightness profiles are from Jorgensen et al. (1992) (solid line) and Strom \& Strom (1978) (dashed line), both in $R$. The high radial velocity of IC 4051 suggests that it is now plunging through the Coma core (see Woodworth \& Harris 2000). This galaxy clearly has a very populous GCS, but it has a spatial concentration remarkably higher than either NGC 4874 or 4889. A more extensive discussion of the comparison between halo light and GCS profile is in Paper III; we con- 
clude similarly that the GCS is slightly shallower for $r \lesssim 15^{\prime \prime}$ but has a steep outer cutoff past $80^{\prime \prime}$ that may follow the halo light.

NGC 4926: The surface brightness profiles are from Jorgensen et al. (1992) (dashed line) and Jansen et al. (2000) (solid line), both in $R$. The GC surface density is far lower than in IC 4051, but both it and the halo light have a similarly steep falloff. We see no strong evidence that the GCS and halo light differ in structure. As we suggested in Paper III, this relatively compact structure and steep outer cutoff may be due to tidal trimming ("harrassment") from the Coma potential well (e.g., Moore et al. 1996), since it is plausible that both IC 4051 and NGC 4926 have oscillated through the Coma core several times during their lifetimes.

NGC 4881: The surface brightness profile is from Thomsen \& Baum (1987) in $\lambda 523 \mathrm{~nm}$. Here we used our data for $V<26.5$, slightly fainter than the normal completeness limit, but we were forced to do this to obtain any kind of estimate of the radial falloff of the GCS. Within the large uncertainties of the GC counts, there is no difference between the GCS distribution and the halo light.

The numerical data for the measured objects as shown in Fig. 6 are summarized in Table 4, in which column (1) gives the mean annulus radius $\langle r\rangle=\sqrt{r_{\text {inner }} \cdot r_{\text {outer }}}$; column (2) the number of objects lying in the annulus before background subtraction, column (3) the annular area in $\operatorname{arcsec}^{2}$ enclosed within the WFPC2 field boundary; and column (4) the number of objects per unit area before background subtraction.

\section{Luminosity Functions}

For NGC 4874, 4889, 4926, and IC 4051, the original exposures in $V$ (though not $I$ ) were deliberately designed to trace the GCLF down to levels as faint as the expected GCLF "turnover point" (peak frequency in number per unit magnitude). We therefore used the $V$ data alone to measure the luminosity functions. A minor loss in using only one band is the inability to reject any candidate objects by color, and although the relative field contamination is quite low at the brighter magnitudes (Fig. (4) compared with the GCs, it increases toward the faint end.

The results for the four galaxies with deepest photometry (i.e. all except NGC 4881) are summarized in Table [5and Figure 7, which show the number of deduced GCs per quarter-magnitude bin, fully corrected for photometric incompleteness and background-subtracted. For NGC 4874 and 4889 , we took all measured objects from $5^{\prime \prime}$ to $120^{\prime \prime}$ from the center of the galaxy to define the GC signal, and used the global background defined above, normalized to the same equivalent area, to subtract off field contamination. For the much more compact IC 4051 system, we used the region from $5^{\prime \prime}$ to $80^{\prime \prime}$ and subtracted off the global background. Finally, for NGC 4926 we took the GC region to be $5^{\prime \prime}-70^{\prime \prime}$ and again used the global background. In Table 5 we also give the LF of the global background population and the total areas of each region are listed in the last line. 
If the GCLFs of the Coma galaxies resemble those found in other giant ellipticals (Harris 2001; Waters et al. 2006), we would expect them to have a Gaussian-like distribution in number per unit magnitude, with a peak frequency (turnover point) roughly at $V^{t o} \simeq 27.7$, and a dispersion $\sigma_{V} \simeq 1.4$. This question is particularly relevant for the two supergiants, because it gives us the opportunity to extend tests for any systematic change of the GC mass distribution function upward to bigger galaxies than we are able to access in Virgo or Fornax. For example, if the supergiants are simply merger products of smaller galaxies that formed all their GCs beforehand, then their product GCLFs should be roughly similar to those in smaller galaxies now. If differences appear, then it may point in turn to a different formation process.

The expected turnover point is within reach of our data, which means that the entire bright half of the GCLF should be visible. For $V \lesssim 27.5$ the photometry in all four galaxy fields is nearly $100 \%$ complete, and comparisons of the GCLFs showed that they are virtually identical in shape over that range, independent of any particular fitting function. An individual puzzling result is for the faint end of the NGC $4889 \mathrm{LF}$, which according to our raw numbers seems to keep rising past the expected turnover point, unlike the other three systems. We have no definite explanation for this except to note that the anomaly occurs just near the photometric completeness limit, where a number of different difficulties with the combined background and completeness corrections can come into play. Conservatively, we have chosen to use only the data for $V<27.75$ for both NGC 4874 and 4889.

Fainter than the expected turnover, the data in the four fields start to differ quite strongly in their completeness functions, and simply adding them up at each magnitude bin would no longer be valid. To make a reasonable estimate of the GCLF shape to slightly deeper levels, we therefore constructed a composite LF by the following procedure, which ensures that we use only the data where the completeness $f_{V}$ is higher than $50 \%$ in each bin:

(a) For $V<27.75$, the composite LF is the direct sum of all four fields, as listed in Table 5, with the individual completeness corrections applied. Over this magnitude range, $f_{V}>0.5$ for all four fields.

(b) For $27.75<V<28.25$, the LF is the direct sum of the NGC 4926 and IC 4051 fields, scaled up by a factor of 3.287 to allow for the fact that these two contribute $30.4 \%$ of the total for $V<27.75$.

(c) For $28.25<V<28.50$, we drop IC 4051 and use only NGC 4926, scaled up by a factor 22.585 .

The result is shown in Figure 8. The explicit assumption we use is that the GCLFs in these four galaxies are intrinsically similar enough to permit this scaling procedure to work. We can test that assumption only over the bright half, but the reason for doing it is to gain the highest statistical confidence we can in carrying the LF past the turnover point. As is also obvious from the scaling procedure just described, the internal uncertainties grow rapidly toward the faint end because of all three factors of increasing field contamination, photometric incompleteness, and scaling factor. If the raw number of objects in the bin is $N$ over area $A$, the number of background objects is $N_{b}$ over area $A_{b}$, the mean photometric completeness factor is $f$, and the population scaling factor is 
$s$, then the fully corrected total in the bin is $N_{G C}=\left(N-N_{b} A / A_{b}\right) s / f$ and its uncertainty due to sampling statistics alone is $\pm\left(N+N_{b}\left(A / A_{b}\right)^{2}\right)^{1 / 2} s / f$. In practice, this can be treated as only a lower limit to the true uncertainty, since the background may have non-Poissonian fluctuations over scale lengths of a few arcminutes.

Next we want to use the combined GCLF to make the best estimate of the turnover magnitude and the intrinsic width or dispersion of the whole distribution. Extensive discussions of interpolating model fits to the GCLF are given in Papers I and III and we will not repeat them at length here. We start by fitting the classic Gaussian function to the binned data in Fig. 8, solving simultaneously for the turnover magnitude $V^{t o}$ and dispersion $\sigma_{V}$, by $\chi^{2}$ minimization. Calculations in a finely spaced grid over a wide range in both parameters shows a well behaved global minimum at $V^{\text {to }}=27.71 \pm 0.07, \sigma_{V}=1.48 \pm 0.04$. This fitted curve is shown in Fig. 8, and the turnover particularly is very close to our original expectation. As discussed in Papers I and III and previous papers on the GCLF (e.g. Secker \& Harris 1993; Hanes \& Whittaker 1987), $V^{\text {to }}$ and $\sigma$ are correlated parameters and tend to be overestimated in cases where the data themselves do not reach clearly past the turnover, since there are no $V>V^{t o}$ observed bins to rule out solutions fainter than the true turnover level. The combined GCLF is based effectively on a residual sample after background subtraction of more than 12,000 GCs.

For comparison, in Paper I we derived $V^{t o}=27.88 \pm 0.10$ for NGC 4874 with a fit constrained to $\sigma_{V} \equiv 1.4$ from an effective sample of $\simeq 2200 \mathrm{GCs}$; while in Paper III we obtained $V^{t o}=$ $27.8 \pm 0.2, \sigma_{V}=1.5 \pm 0.1$ for IC 4051 from an effective sample of $\simeq 2000$ GCs. In both cases, the background was a "local" one defined from the outer regions of each field itself, and thus for NGC 4874 the GC population was somewhat oversubtracted. The solution for the turnover level and dispersion in the present paper is based on a GC sample five times larger than in either of these previous studies, as well as an improved background definition. Both of these factors mean that we can constrain the solution much better than in our previous work. It is worth noting as well that our combined Coma sample of $\sim 10^{4}$ GCs brighter than the GCLF turnover is more than twice as large as the numbers measured in all the Virgo galaxies combined (Peng et al. 2006; Jordán et al. 2007).

The Gaussian is by far the most frequently used shorthand description of the GCLF, but has no physical basis. Other, more physically based, models have been proposed for the GCLF that combine aspects of numerical convenience with some foundation in a plausible initial mass function for GCs and its subsequent dynamical evolution. Descriptions of the LF in its alternate form as number per unit luminosity $(d N / d L$, the luminosity distribution function or LDF) connect much more directly with the mass distributions of young star clusters, and began to be applied to globular cluster populations in several galaxies some years ago (e.g. Harris \& Pudritz 1994; McLaughlin 1994). Schechter-like functions have been applied to the high-mass end of the globular cluster distribution by, e.g., Burkert \& Smith (2000), and to the observed distributions for young massive clusters by Gieles et al. (2006), Whitmore et al. (1999), and Whitmore et al. (2002) among others. A similar form has been developed further into an evolved Schechter function formulation 
(Fall \& Zhang 2001; Jordán et al. 2006, 2007) which accounts roughly for the long-term dynamical evolution of GCs and the preferential loss of low-mass clusters over time. Jordán et al. (2007) have tested this model extensively against the database from the Virgo cluster survey over a wide range of host galaxy luminosities. Strictly on numerical grounds, this function is better able to match the asymmetry of the complete GCLF: in the number-vs.-magnitude plot, the faint end past the turnover is observed to have a broader wing than the bright half. However, for our purposes of defining the turnover point, this feature of the model is irrelevant, since the Coma data fall far short of the luminosity limit at which the differences between this function and the simpler Gaussian become important. In Fig. 8, we show our best-fit evolved Schechter function in the form expressed by Jordán et al. (2007),

$$
N(V)=\operatorname{const} \frac{10^{-0.4\left(V-V_{c}\right)}}{\left[10^{-0.4\left(V-V_{c}\right)}+10^{-0.4\left(\delta-V_{c}\right)}\right]^{2}} \exp \left[-10^{-0.4\left(V-V_{c}\right)}\right] .
$$

Our numerical solution gives $\left(\delta-V_{c}\right) \simeq 3.20$ and $V_{c} \simeq 24.40$, both uncertain to \pm 0.05 mag. As is evident from Fig. 8, there is no distinguishable difference between the best-fit Gaussian and Schechter-like functions for the luminosity range around the turnover point and brighter. This bright-half regime is what our data cover. It is only on the unobserved fainter half of the GCLF that the differences between the two analytical approximations show up strongly. For the Schechter function fit, the magnitude $V_{c}$ above which the bright-end exponential cutoff begins to dominate the shape of the LF corresponds roughly to a cluster mass $M_{c} \simeq 3 \times 10^{6} M_{\odot}$ for $(M / L)_{V} \simeq 2$ (McLaughlin 2000; Rejkuba et al. 2007).

The cutoff mass that we find is noticeably higher, and the overall LF broader, than in any of the Virgo galaxies. However, Jordán et al. (2007) already show from the Virgo sample that $M_{c}$ increases systematically with host galaxy luminosity. Since our Coma GCS sample is dominated by the supergiants NGC 4874 and NGC 4889, which are more luminous than anything in Virgo, an extrapolation upward from their correlation of $M_{c}$ vs. $M_{B}^{t}$ puts the Coma result quite close to what that trend would predict.

For our fiducial Coma distance $(m-M)_{0}=34.97 \pm 0.13$, then the observed turnover $V^{t o}=$ $27.71 \pm 0.07$ converts to $M_{V}^{t o}=-7.32 \pm 0.13$, subtracting $A_{V}=0.03$ and a $V$-band K-correction of 0.03 (see Paper I and Frei \& Gunn 1994). This turnover luminosity agrees extremely well with the mean from many other giant ellipticals in Virgo, Fornax, and other nearby groups of $M_{V}^{t o}=-7.3 \pm 0.1$ (e.g. Harris 2001, and Paper I). Phrased differently, if we had chosen here to use $M_{V}^{t o}$ for its classic purpose as a standard candle (Jacoby et al. 1992; Harris 2001) and to derive the distance to Coma, as we did in Paper I, we would obtain $(m-M)_{V}=35.01 \pm 0.15$. The corresponding Hubble ratio would be $h=0.73 \pm 0.07$. Most of the uncertainty in this case is actually from the local calibration of the turnover luminosity.

Perhaps the most relevant individual comparison is with M87 alone, the central Virgo giant, whose GCS has been extensively surveyed to very deep levels in $(V, I)$. (A larger composite sample is available from the Virgo cluster survey of Jordán et al. (2007), but these are in the $z^{\prime}$ band.) 
Waters et al. (2006) present a GCLF from WFPC2 data in the same $V$ and $I$ bands as we use here, which extends well past the turnover level to high completeness. By subdividing their data into radial bins, they show that the LF is also independent (to within the statistical uncertainties) of location, so we use their full data sample read from their Figure 4. We normalize the M87 data to the same total GC population brighter than the turnover level, which for M87 they find to lie at $V^{t o}(M 87)=23.60$ with $\sigma_{V}=1.42$ for a Gaussian fit; since we measure $\simeq 9000$ GCs to that level in the combined Coma 4-galaxy sample, and they find $\simeq 519$ GCs to the turnover in M87, we multiply their bin totals by 17.5. For an adopted Virgo distance of $16 \mathrm{Mpc}$ (Paper I) and $A_{V}=0.03$, this gives a relative distance modulus $\Delta m=4.04$ mag (again adding the 0.03-mag K-correction at Coma). Thus displaced to the Coma distance, the M87 GCLF turnover would appear at $V^{t o} \simeq 27.64$, in good agreement with our data. The normalized M87 distribution is also shown in Fig. 8, with 19 times fewer GCs contained in the sample relative to the Coma data, it shows stochastic differences from bin to bin that are distinctly larger, but there are no clear systematic differences. A standard Kolmogorov-Smirnov two-sample test shows that there is a negligible probability that they are intrinsically different for any magnitude range brighter than the turnover region.

Lastly, our best-fitting GCLF dispersion of $1.48 \pm 0.04$ mag fits into the general pattern that the dispersion increases systematically with parent galaxy luminosity (Jordán et al. 2006, 2007). Our composite sample corresponds roughly to $M_{B}^{t}=-22.4$, the average of NGC 4874 and 4889. The mean relation $\sigma \simeq 1.12-0.093\left(M_{B}^{t}+20\right)$ derived by Jordán et al. (2006) would then predict $\sigma=1.34$ for our sample. This is smaller than what we observe, but the difference is within the galaxy-to-galaxy scatter around the mean relation, which is at least \pm 0.1 mag (see their Figure 1).

The two main conclusions from our LF analysis are that (1) the GCLF turnover luminosity is very similar to those in other giant ellipticals; and (2) the bright half of the GCLF may be significantly broader than in the Virgo members, as indicated by either the Gaussian dispersion $\sigma$ or the Schechter-function parameter $\left(\delta-m_{c}\right)$. We suggest that this evidence favors a formation process in which the supergiants did not assemble just as "dry mergers" of previously built systems. Because the GCLF is broader, the supergiants have relatively more high-mass GCs that seem more likely to have been built in the gas-rich stages of hierarchical merging. If GCs had simply been added later from gas-free satellite accretions, it is difficult to see how the very highest-mass end of the GCLF could have been populated to the extent that we see.

\section{Total Populations and Specific Frequency}

Knowing the results from the GCLF analysis and the radial profile information, we can next estimate for each galaxy the total globular cluster population $N_{t}$ and specific frequency, $S_{N} \equiv$ $N_{t} \cdot 10^{0.4\left(M_{V}^{t}+15\right)}$ (Harris \& van den Bergh 1981) where $M_{V}^{t}$ is the integrated magnitude of the galaxy. To estimate $N_{t}$, we integrate under the GCS radial profiles from Fig. 6 and then multiply that total by the fraction of the GCLF that is fainter than the observed limit. Individual details 
follow.

$N G C$ 4874: Of the five galaxies in our study, this is by far the hardest for which to calculate a global $S_{N}$. To estimate the total GC population, we use the radial range $4^{\prime \prime}<r<150^{\prime \prime}$ (almost the entire area of the WFPC2 field) and conservatively take the magnitude range $V<26.5$ for which the photometry is complete. We further color-select to include objects in the range $0.5<(V-I)<1.8$. The background number density in this magnitude and color range, as determined from the "global" background field, is $\sigma_{b k g d}=0.011 \mathrm{arcsec}^{-2}$. Over $4^{\prime \prime}<r<150^{\prime \prime}$, integration of the GC density profile gives $3875 \pm 468$ clusters after background subtraction. Given that the innermost profile is nearly flat (cf. Fig. 5), we use the value of $\sigma_{G C}$ at $4^{\prime \prime}$ and add 15 more GCs for the region inside that, giving $N \simeq 3890 \pm 470$ GCs within $r=150^{\prime \prime}$ of galaxy center. Next, we use the standard GCLF Gaussian shape determined in the previous section to extrapolate the total over all magnitudes. The cutoff $V_{\text {lim }}=26.5$ is $0.82 \sigma$ short of the GCLF turnover at $V=27.71$, which means that we multiply our observed GC total by 4.81 to obtain the GC population over all magnitudes (we note here that the specific frequency $S_{N}$ is defined assuming a Gaussian GCLF: that is, we find the number of observed GCs to the turnover point, double that, and use the resulting total to calculate $S_{N}$ ). This estimate yields $N_{t}=18700 \pm 2260$. With $M_{V}^{t}=-23.43$, we obtain $S_{N}=7.9 \pm 1.0$. This value supersedes our original (lower) value in Paper II. The increase results from a combination of a lower adopted background level and an 0.15-mag correction to the $V$ magnitude zeropoint for this galaxy (see Section 6 below). Clearly, the best way to refine the measurement of $N_{t}$ further is to obtain wider-field data and a better global background.

This estimate must, however, be only a lower limit to the true GC population because it does not account for the numbers of clusters that must be present outside $r=150^{\prime \prime}(\sim 72 \mathrm{kpc})$. The obvious indication from Fig. 6 is that the GC density profile continues well beyond. We do not know how far to integrate outward, but it should continue at least twice this far, to $r \sim 300^{\prime \prime}(143$ $\mathrm{kpc})$; for comparison, the halfway point to NGC 4889 is at $205^{\prime \prime}(100 \mathrm{kpc})$. The $r^{1 / 4}$ profile shown in Fig. [6 for the outer region $r \gtrsim 20^{\prime \prime}$ is roughly given by $\log \sigma_{c l}=-0.4912 r^{1 / 4}+0.2377$ where $r$ is in arcseconds and $\sigma_{c l}$ is in number of GCs per $\operatorname{arcsec}^{2}$. Simply extrapolating this outward to $r=300^{\prime \prime}$ as it is (which we have no obvious reason not to do) would increase the total GC population by a factor of $\simeq 2.2$; that is, there are more GCs outside $r=150^{\prime \prime}$ than inside if the same profile holds true. We would then have to increase our estimate of the total GCS to $N_{t} \sim 41,000$ and the specific frequency to $S_{N} \sim 17$. Specific frequency values this high are certainly within the range of measurements for other cD-type galaxies (see, for example, Wehner et al. 2008; Tamura et al. 2006).

It is probably safe to say that NGC 4874 contains more than 30,000 GCs, making it the most populous globular cluster system that we know of anywhere in the local universe. A more quantitative estimate through wider-field imaging would be an extremely interesting extension of the current study, and would also permit exploration of the GC population within the Coma core region at large. 
NGC 4889: For the second of the two supergiants, the GC radial density curve is steeper. For this reason, it approaches much more closely to the background (Fig. 6) at the edges of the WFPC2 field, and so the the estimate of $N_{t}$ can converge reasonably. Using $V<27$ and the global background, we obtain $\sigma_{b}=0.040$ and a total of $3200 \pm 400$ clusters for radii $4^{\prime \prime}<r<150^{\prime \prime}$, and add another $\simeq 50$ for $r<4^{\prime \prime}$. Extrapolating over all magnitudes gives $N_{t}=11000 \pm 1340$. The outward extrapolation to account for clusters beyond the observed field is a much smaller correction than for NGC 4874: we find with $r(\max )$ the same as used for NGC 4874 above that it is necessary to increase $N_{t}$ by at most $10-15 \%$, i.e. $N_{t} \simeq 12000 \pm 2000$. With $M_{V}^{t}=-23.54$ we have $S_{N}=4.7 \pm 0.6$. This result is squarely in the normal range established by the giants in Virgo and Fornax; its huge total GC population simply goes along with its very high luminosity.

IC 4051: This GC-rich system fortunately has an even steeper radial profile, so the limits on $N_{t}$ are even better. With the same magnitude limit and background as above, we obtain $2000 \pm 160$ for $4^{\prime \prime}<r<115^{\prime \prime}$, and we need to add only about 10 more for the innermost region. Integrating over all magnitudes gives $N_{t}=6700 \pm 530$. The radial profile is so close to background at this radius that we do not add any further corrections; thus, with $M_{V}^{t}=-21.85$ we have $S_{N}=12.1 \pm 1.0$, quite similar to our result in Paper III. Again we see the importance of normalizing $N_{t}$ to the galaxy luminosity. By sheer numbers, the two central supergiants may contain most of the GCs in the entire Coma cluster, but IC 4051 appears to have been at least as efficient per unit luminosity as NGC 4874 and various $\mathrm{cD}$ galaxies elsewhere in forming them.

NGC 4881: For $V<26.0$ there are $\simeq 25$ GCs after background subtraction and within radii $3^{\prime \prime}<r<30^{\prime \prime}$. To these we add about $25 \%$ more as an estimate of the number of GCs lying in the innermost and outermost regions $\left(r<3^{\prime \prime}, r>30^{\prime \prime}\right)$ not covered by our data. Then, to correct the total for the GCs fainter than our adopted limit of $V=26$, we note that this limit is 1.73 mag or 1.23 standard deviations brighter than the mean GCLF turnover (previous section). This range includes $11 \%$ of the true total cluster population. We therefore scale up the observed number by a factor of 9 . With $M_{V}^{t}=-21.46$ (Table 1) we then obtain $S_{N}=0.77 \pm 0.21$, in very good agreement with the earlier estimate of $S_{N} \sim 1.0$ by Baum et al. (1995). This strikingly low- $S_{N}$ value is at the bottom end of the observed range for any type of E galaxy, regardless of luminosity or environment. Our value may in fact even be a slight overestimate if the galaxy actually has no clusters beyond the $30^{\prime \prime}(15 \mathrm{kpc})$ radius.

NGC 4926: Again for the same limits as used above, we calculate for NGC 4926 that for $4^{\prime \prime}<$ $r<100^{\prime \prime}$ there should be $(570 \pm 110)$ clusters, and add in $\sim 30$ more clusters for the innermost $4^{\prime \prime}$. Extrapolating the radial profile outward gives at most 140 more GCs, thus $N_{t}=1300 \pm 300$ over all magnitudes as well. With $M_{V}^{t}=-22.03$ we then have $S_{N}=2.0 \pm 0.5$. This total, on the low-to-normal side for E galaxies (Harris 2001), is 6 times smaller than for IC 4051 but three times larger than in NGC 4881.

Our final $N_{t}$ and $S_{N}$ estimates are listed in Table 1. A completely independent set of specific frequency measurements for the Coma galaxies has been made by Marin-Franch \& Aparicio (2002) 
through surface brightness fluctuations. We have three galaxies in common: for NGC 4874, 4889, and IC 4051 they derive $S_{N}=(9.0 \pm 2.2),(4.0 \pm 1.2)$, and $(12.7 \pm 3.2)$. These numbers are in good agreement with our findings, to well within their combined internal uncertainties. It is also worth

noting that when the Blakeslee \& Tonry (1995) results are adjusted for a $10 \%$ larger Coma distance to bring them in line with the current distance, their total populations would become $\simeq 20700$ in NGC 4874 within $175^{\prime \prime}$ and $\simeq 15800$ in NGC 4889. These are both consistent with our estimates for the totals within $150^{\prime \prime}$.

\section{Color and Metallicity Distributions}

Lastly, we discuss the magnitude/color distributions a bit further for the GCs in all five Coma galaxies. Specifically, we investigate whether or not the mean colors and color distribution fall into the now well-established pattern of bimodality (e.g. Zepf \& Ashman 1993; Larsen et al. 2001; Peng et al. 2006; Harris et al. 2006; Kundu \& Zepf 2007; Strader et al. 2007, among many others). Although the $(V-I)$ index is not very sensitive to metallicity (see Gebhardt \& Kissler-Patig 1999; Kundu \& Whitmore 2001; Larsen et al. 2001), such data for many galaxies show a virtually universal pattern whereby the metal-poor mode is near $\langle V-I\rangle_{0} \simeq 0.95$ and the metal-richer mode is near $\langle V-I\rangle_{0} \simeq 1.15$, with a weak trend for both modes to become redder with galaxy luminosity Larsen et al. 2001; Strader et al. 2004; Brodie \& Strader 2006) more or less as $Z \sim L^{0.15}$. We would thus expect the GC modes for the Coma supergiants particularly to be among the reddest known.

In Paper II we claimed that the mean colors for the GCs in NGC 4874 were bluer than normal for a giant elliptical, suggesting that most of its cluster population was fairly metal-poor. However, we discovered during the data reductions for the present paper that the color scale of our photometry in Paper II was wrong by $\sim 0.15$ magnitude, resulting from the accidental use of an incorrect color coefficient in the transformation from $F 606 W$ to $V$. In the present paper, our NGC 4874 data (and indeed all the other galaxies) result from completely independent new reductions starting from the raw images, and have all used the correct transformations. As will be seen below, the true color distribution for the NGC 4874 clusters indicates a normal combination of metal-poor and metal-rich clusters.

The color-magnitude data in Fig. 4 show that the raw $(V-I)$ colors fall in the normal range for GCs in giant E galaxies, except for NGC 4881 in which the GCs appear to be almost entirely blue. In the other four, the standard red and blue modes (if present) are substantially blurred and overlapped by photometric measurement scatter. We restrict our discussion of the color histograms to the relatively bright range $I<25.5$, well above the GCLF turnover point. These are shown in Figure 9 .

For the three most populous GCSs, we can go a bit further and look at the same histograms broken down by magnitude range, as shown in Figure 10, The numbers of stars in the global 
background field in the same ranges, normalized to the same area as each galaxy field, are shown in the shaded histograms. In order to minimize the damaging effects of both field contamination and color spread from photometric measurement scatter, we choose to use only the objects in the very bright range $I<24.5$ (corresponding roughly to GC luminosities $M_{V} \lesssim-9.5$ ) for further analysis. After subtracting off the small amount of background, we obtain the histograms in Figures 11, 12, and 13, which show our best estimates for the residual color distributions of the GCs alone.

Do these distributions show the now-standard bimodal form? Even with our most rigorous subselection of data, the answer is ambiguous, and in the end we can gain only some hints. We use the statistical package RMIX (MacDonald 2007; Wehner et al. 2008) to perform multimodal fitting to each histogram, and specifically to gauge whether or not two modes might be present. Although RMIX can use a variety of fitting functions, we stick with the Gaussian model for simplicity and ease of comparison with the previous literature. (See Wehner et al. 2008 for examples of its use in the same context of GCS color distributions.) In the left panels of Figs. 11, 12, and 13, we show the best-fit unimodal Gaussian curve for each of the three galaxies, with means and standard deviations listed in Table 6. In all cases, these provide at least roughly adequate descriptions of the run of the data. If we next ask RMIX to find two modes with complete freedom to solve for the means, standard deviations, and proportions, the code tends to converge to solutions not far from the single-Gaussian solution, i.e. ones in which one of the modes takes up $\lesssim 10 \%$ of the population. In short, without external arguments to the contrary, we have no compelling evidence to claim the presence of bimodal sequences.

Instead, we ask a slightly more restricted question. If the red and blue modes are actually present and simply being obscured by photometric scatter, we would like to know most importantly their mean colors $\mu_{1}, \mu_{2}$ and the proportions $p_{1}, p_{2}$. To estimate these, we can press the data a little further by performing constrained bimodal fits where we assume fixed input values for various combinations of the parameters, such as the dispersions $\sigma_{1}, \sigma_{2}$. We have experimented widely with various combinations of these constraints, which are straightforward to do within RMIX. Samples of these are shown in the right panels of Figs. 11, 12, and 13. These are not intended to be our "best" choices because ones producing equally good fits can be obtained with other pairs of parameters (although we justify our choice of the 30/70 proportions a bit further below). They are, however, illustrative of the quality of fit that the constrained bimodal solutions provide. Any other solutions in which either $p_{1} \sim p_{2}$ or $\sigma_{1} \sim \sigma_{2}$ yield quite similar means $\mu_{1}, \mu_{2}$ to the ones shown here; that is, the solutions for the mean colors tend to be robust against plausible changes in either the proportions or dispersions. The sample two-Gaussian solutions are listed in Table 6 for these three galaxies, in the pair of lines just below the single-Gaussian data. In each case the double-Gaussian solution is slightly but not strongly preferred over the single-Gaussian.

Perhaps the most interesting single conclusion from these admittedly rough tests is that the deduced mean color of the blue mode is virtually identical at $\left\langle\mu_{1}\right\rangle=0.98 \pm 0.02$ in all three systems. The same is true for the red mode, where $\left\langle\mu_{2}\right\rangle \simeq 1.15 \pm 0.02$ in all three systems. Subtracting off the foreground reddening of just $0.01 \mathrm{mag}$, and a K-correction $K_{V}-K_{I} \simeq 0.02$ (e.g. Frei \& Gunn 1994), 
we have $\mu_{0}$ (blue $)=0.95, \mu_{0}($ red $)=1.12$, which are quite close to the previously known values for the brightest giant ellipticals elsewhere (Larsen et al. 2001; Strader et al. 2004; Brodie \& Strader 2006) and agree with these to well within the uncertainties of the data. These two arguments reinforce our tentative conclusion that the normal two modes may be present in these Coma members, and lie at least approximately at the expected metallicity values.

One further test of these three color distributions, now by radius, is shown in Figure 14. If two subpopulations of GCs are present in blue and red sequences, then evidence from other galaxies indicates that we may expect to find an overall population gradient with galactocentric distance in the sense that the redder, more metal-rich GCs are more centrally concentrated. As a rough dividing line, we simply take GCs brighter than $I=25$ with $(V-I)<1.05$ and put them into the "blue" population, and ones with $I<25,(V-I)>1.05$ into the "red" population. Fig. 14 shows the result of two numerical experiments. In the first panel we show the mean color of the red and blue subsamples in radial bins. We conclude that no net change in the mean color of either group is present. (As noted above from the bimodal Gaussian fits, the mean colors of each group are also closely similar in all three galaxies.)

In the second panel we show the population ratio $N($ red $) / N($ blue $)$ in the same radial bins. A slight but noticeable trend appears in all three galaxies in the expected sense, for the more metal-rich clusters to dominate more in the inner regions. This trend is most noticeable for NGC 4889 (dotted line) but for $r>30^{\prime \prime}$ it is present almost equally in all three. Overall the red ones make up more than $2 / 3$ of the total (justifying post facto our choice of the $30 / 70$ proportions in the Gaussian fits described above). However, at very large radius (40 kpc and more) the ratio begins to approach $N($ blue $) \simeq N($ red $)$. We add this evidence to support our tentative conclusion that a radial population gradient does exist in these galaxies: the mean metallicities of both the two modes are roughly constant with radius, but their relative numbers change. Very similar evidence has been presented for giant ellipticals in Virgo, Fornax and other Abell clusters (Geisler et al. 1996; Dirsch et al. 2003; Rhode \& Zepf 2004; Harris et al. 2006).

In all three of NGC 4874, 4889, and IC 4051 we find that more than half the clusters fall in the metal-rich mode at any radius within our surveyed area. If these proportions - which we emphasize are admittedly still internally uncertain - are confirmed by more precise data where the two modes can be split more definitively, it is worth noting that they would disagree with the trend noted by Peng et al. (2008). For the Virgo galaxies, Peng et al. find essentially that the ratio $N($ red $) / N($ blue $)$ increases steadily as we pass along the sequence from dwarfs to giants, reaching a ratio near $\sim 0.8$ for moderately large ellipticals (see their Figs. 8 and 9 ). But the ratio then starts to decrease again down to $\simeq 0.5$ or less for the biggest Virgo giants (M87, M49 and a few others). Our three Coma supergiants, for which we find tentative evidence that $N($ red $) / N($ blue $) \gtrsim 1$, may provide new counterexamples to this trend, perhaps suggesting that at the very densest and most massive protogalactic environments, efficient and high-metallicity cluster formation was especially favored. To put this result another way, whereas Peng et al. (2008) found that the galaxy-togalaxy variations in specific frequency in Virgo are driven mostly by the numbers of blue clusters, 
the similarly high specific frequencies in the Coma supergiants are produced mostly by their large numbers of red clusters.

One final note about the color distributions relates to their behavior at the bright end. Wehner et al. (2008) find, for the Hydra cD galaxy NGC 3311, that the red GC sequence extends distinctly further upward than does the blue sequence. The very brightest red GCs extend well into the UCD-type luminosity regime, suggesting a connection between the most massive GCs and UCDs. In our Coma data, we see a hint of such a feature in the color-magnitude diagrams for NGC 4874 and IC 4051 (Fig. 4). For $I<23$ (corresponding to $M_{I}<-12$ ), much more than half the GCs are on the red side at $(V-I) \simeq 1.2$. More extensive areal coverage of the NGC 4874 system particularly should reveal whether or not this effect is real, or simply an accident of small-number statistics. In addition, a larger sample of red, very luminous clusters would allow an interesting test of the recent model by Bailin \& Harris (2008), which predicts that a modest but real increase of cluster metallicity with mass should appear at this top end. This mass/metallicity relation (MMR) in their model is driven by self-enrichment during cluster formation and should affect the highest-mass clusters along both the blue and red sequences, but is more noticeable along the blue sequence (Harris et al. 2006; Strader et al. 2006; Mieske et al. 2006) because initial (pre-enrichment) metallicity level is much lower.

For NGC 4881 and 4926, the GC populations are too small to attempt more than singlemode descriptions. In Figure 15 we show the results for these two low $-S_{N}$ systems, with numerical solutions as listed in Table 6. Guided by the radial density plots in Fig. 5, we used local background subtraction to define the residual color histograms for these two systems. For NGC 4881, the local background was adopted to be the region $r>40^{\prime \prime}$ from galaxy center, while for NGC 4926 it was $r>50^{\prime \prime}$.

The color distribution for NGC 4881 is perhaps the most surprising one in our entire study, falling farther away from the normal pattern than any of the others in our sample. The few GCs that it has are blue, with a mean color $\mu=0.79$ and rather narrow histogram. The correlation of blue-sequence $(V-I)$ color with galaxy luminosity by Strader et al. (2004) would predict $\mu \simeq 0.94$, far redder than what we see; mean colors as low as $\simeq 0.8$ are typically found only in dwarf ellipticals or in the halos of spirals like the Milky Way. Both the low $S_{N}$ of this galaxy and its very metal-poor GC population are severe challenges to normal models for formation of large ellipticals. If it did form through any series of mergers, these must have been virtually gas-free to avoid making any more metal-richer clusters, but furthermore, the progenitor systems must have been lacking such clusters to begin with. But if the original mergers of small progenitors were that gas-free, it would have been even more difficult to form the metal-rich field stars that make up its bulk population. The mean color of the galaxy as a whole of $(B-V)) 0 \simeq 1.00$ is in the normal range for metal-rich gE's (see also Thomsen \& Baum 1987). This remains an intriguing system.

Finally, NGC 4926 presents almost the opposite case to puzzle over. The color histogram is fairly broad and has a mean color $\mu=1.19$ that is a bit redder than even the red sequences 
discussed above for the supergiants. The small excess of very red objects with $(V-I)>1.5$ is mostly clustered in one quadrant of the WFPC2 image and probably is due to a background cluster of galaxies. If there is a blue GC sequence (perhaps centered at $(V-I) \simeq 0.95$; see Fig. 15) it would resemble the blue modes we found for the three major systems above, though the statistics are too uncertain to allow firm claims. Rapid early mergers, at a time when a large amount of gas was available for star formation, might be capable of producing a GC abundance distribution like this, but it is less obvious how the same series of events could have left behind a rather low total population of clusters.

We will leave the discussion at this point. Much improvement in understanding the GC metallicity distributions in these galaxies can be expected from ACS/WFC imaging in the near future (Carter et al. 2008).

\section{Summary}

In this paper, the last of our series on the Coma galaxies, we have presented our analysis of WFPC2 imaging of the globular cluster systems around five giant ellipticals. Our principal results can be summarized as follows:

- The supergiant cD galaxy NGC 4874 holds perhaps more than 30 thousand clusters; its true radial extent is not yet known but may fill up a substantial part of the Coma core. It is the most populous globular cluster system in any galaxy that we know of. Nevertheless, the radial structure analysis shows that its GCS belongs to the galaxy rather than the Coma potential well as a whole (cf. Paper II).

- The range of specific frequencies we see in just these five galaxies is amazingly large, ranging from a low of $S_{N}<1$ in NGC 4881 up to $S_{N} \simeq 12$ in both NGC 4874 and IC 4051. Their past histories of tidal truncation, gas-free or gas-rich mergers, or GC formation efficiency may all have played roles, but puzzles remain that simply do not have clear explanations as yet.

- Our $V$-band data for four galaxies (NGC 4874, 4889, 4926, IC 4051) reach deep enough to allow us to study the bright half of the GC luminosity function thoroughly, and to define the classic turnover point with some confidence. We find $V^{t o}=27.71 \pm 0.07$, corresponding to $M_{V}^{t o}=-7.3$ and quite similar to most normal E galaxies. Our definition of the GCLF is based almost $10^{4}$ GCs brighter than the turnover point. We find that both a simple Gaussian curve and an "evolved Schechter function" fit these bright parts of the GCLF equally well. Though the turnover luminosity is the same as in many other giant ellipticals, the GCLF shape is noticeably broader, extending to quite high GC mass.

- For the three biggest GC systems (NGC 4874, 4889, IC 4051), analysis of the $(V-I)$ color distributions shows that all three populations are dominated by red, metal-rich clusters. 
Finer analysis is hampered by the random scatter in the photometry. However, various tests including bimodal fitting to the color histograms, and measurement of the population ratios (red vs. blue GCs as a function of radius), show clear hints that the two normal color modes exist in these three systems, at mean colors $\langle V-I\rangle_{0}$ (blue) $\simeq 0.95$ and $\langle V-I\rangle_{0}$ (red) $\simeq 1.12$. These values fall along the previously established correlations of mean color with galaxy luminosity.

- The three non-supergiant ellipticals in our study, NGC 4881, NGC 4926, and IC 4051, present opposing challenges to understanding their formation. NGC 4881 has few GCs and these have entirely blue colors like those in dwarf ellipticals; it is completely lacking the metal-rich GCs that we conventionally find in other big galaxies of all types. NGC 4926 also has a low $S_{N}$, and is far from the most luminous Coma member, but is dominated by GCs that are at least as red as those in the supergiants. Finally, IC 4051 has a $S_{N}$ value as high as many cD-type central giants (and more than an order of magnitude larger than in NGC 4881), yet is structurally quite similar in other ways to NGC 4881 and 4926.

No single formation scenario seems to be able to account for the huge range of characteristics we see in all of these GCSs.

This research was supported through grants to WEH, DAH, and CJP from the Natural Sciences and Engineering Research Council of Canada. We are grateful to John Blakeslee for a careful and constructive reading of the first version of the paper. 


\section{REFERENCES}

Adami, C., Biviano, A., Durret, F., \& Mazure, A. 2005, A\&A, 443, 17

Baier, F.W., Fritze, K., \& Tiersch, H. 1990, Astr.Nachr.311, 89

Bailin, J., \& Harris, W.E. 2008, ApJ, submitted

Baum, W.A. et al. 1995, AJ, 110, 2537

Baum, W.A. et al. 1997, AJ, 113, 1483

Blakeslee, J. P. 1999, AJ, 118, 1506

Blakeslee, J. P., \& Tonry, J.L. 1995, ApJ, 442, 579

Blakeslee, J. P., Tonry, J.L., \& Metzger, M.R. 1997, AJ, 114, 482

Bower, R.G., Lucey, J.R., \& Ellis, R.S. 1992, MNRAS, 254, 589

Brodie, J.P., \& Strader, J. 2006, ARAA, 44, 193

Burkert, A., \& Smith, G.H. 2000, ApJ, 542, L95

Carter, D. et al. 2008, ApJS, 176, 424

Colless, M., \& Dunn, A.M. 1996, ApJ, 458, 435

Dirsch, B., Richtler, R., Geisler, D., Forte, J.C., Bassino, L.P., \& Gieren, W.P. 2003, AJ, 125, 1908

Evstigneeva, E.A., Drinkwater, M.J., Peng, C.Y., Hilker, M., DePropris, R., Jones, J.B., Phillipps, S., Gregg, M.D., \& Karick, A.M. 2008, AJ, 136, 461

Fall, S.M., \& Zhang, Q. 2001, ApJ, 561, 751

Fitchett, M., \& Webster, R. 1987, ApJ 317, 653

Forbes, D. A., Brodie, J. P., \& Grillmair, C. J. 1997, AJ, 113, 1652

Freedman, W. et al. 2001, ApJ, 553, 47

Frei, Z., \& Gunn, J.E. 1994, AJ, 108, 1476

Gebhardt, K., \& Kissler-Patig, M. 1999, AJ, 118, 1526

Geisler, D., Lee, M.G., \& Kim, E. 1996, AJ, 111, 1529

Geiles, M., Larsen, S.S., Bastian, N., \& Stein, I.T. 2006, A\&A, 450, 129

Gurzadyan, V.G., \& Mazure, A. 2001, NewA, 6, 43 
Hanes, D.A., \& Whittaker, D.G. 1987, AJ, 94, 906

Harris, W.E. 1987, ApJ, 315, L29

Harris, W.E. 1996, AJ, 112, 1487

Harris, W.E. 2001, in Star Clusters, Saas-Fee Advanced Course 28 (New York: Springer), ed. L.Labhardt \& B.Binggeli.

Harris, W.E., Allwright, J.W.B., Pritchet, C.J., \& van den Bergh, S. 1991, ApJS, 76, 115

Harris, W.E., Kavelaars, J.J., Hanes, D.A., Hesser, J.E., \& Pritchet, C.J. 2000, ApJ, 533, 137 (Paper II)

Harris, W.E., \& Pudritz, R.E. 1994, ApJ, 429, 177

Harris, W.E., \& van den Bergh, S. 1981, AJ, 86, 1627

Harris, W.E., Whitmore, B.C., Karakla, D., Okoń, W., Baum, W.A., Hanes, D.A., \& Kavelaars, J.J. 2006, ApJ, 636, 90

Jacoby, G.H., Branch, D., Ciardullo, R., Davies, R.L., Harris, W.E., Pierce, M.J., Pritchet, C.J., Tonry, J.L., \& Welch, D.L. 1992, PASP, 104, 599

Holtzman, J. et al. 1995, PASP, 107, 1065

Jansen, R.A., Franx, M., Fabricant, D., \& Caldwell, N. 2000, ApJS, 126, 271

Jordán, A. et al. 2006, ApJ, 651, L25

Jordán, A., et al. 2007, ApJS, 171, 101

Jorgensen, I., Franx, M., \& Kjaergaard, P. 1992, AApS, 95, 489

Kavelaars, J. J., Harris, W. E., Hanes, D. A., Hesser, J. E., \& Pritchet, C. J. 1999, ApJ, 533, 125 (Paper I)

Kundu, A., \& Zepf, S.E. 2007, ApJ, 660, L109

Kundu, A., \& Whitmore, B.C. 2001, AJ, 121, 2950

Larsen, S.S. 1999, A\&AS, 139, 393

Larsen, S.S., Brodie, J.P., Huchra, J.P., Forbes, D.A., \& Grillmair, C.J. 2001, AJ, 121, 2974

Larson, R. B. 1993, in The Globular Cluster - Galaxy Connection, ASP Conf. Ser. 48, edited by G. H. Smith \& J. P. Brodie (San Francisco: ASP), 675

MacDonald, P.D.M. 2007, documentation and code at http://www.math.mcmaster.ca/peter/mix/mix.html, Department of Mathematics and Statistics, McMaster University 
Marin-Franch, A., \& Aparicio, A. 2002, ApJ 568, 174

McLaughlin, D.E. 1994, PASP, 196, 47

McLaughlin, D.E. 2000, ApJ, 559, 812

Merritt, D., \& Tremblay, B. 1994, AJ, 108, 514

Mieske, S. et al. 2006, ApJ, 653, 193

Moore, B., Katz, N., Lake, G., Dressler, A., \& Oemler, A. Jr. 1996, Nature, 379, 613

Neumann, D.M., Lumb, D.H., Pratt, G.W., \& Briel, U.G. 2003, A\&A, 400, 811

Peletier, R.F., Davies, R.L., Illingworth, G.D., Davis, L.E., \& Cawson, M. 1990, AJ, 100, 1091

Peng, E.W. et al. 2006, ApJ, 639, 95

Peng, E.W. et al. 2008, ApJ, 681, 197

Rejkuba, M., Dubath, P., Minniti, D., \& Meylan, G. 2007, A\&A, 469, 147

Rhode, K.L., \& Zepf, S.E. 2004, AJ, 127, 302

Secker, J., \& Harris, W.E. 1993, AJ, 105, 1358

Spergel, A.N. et al. 2007, ApJS, 170, 377

Stetson, P.B. 1994, PASP, 106, 250

Strader, J., Beasley, M.A., \& Brodie, J.P. 2007, AJ, 133, 2015

Strader, J., Brodie, J.P., Spitler, L., \& Beasley, M.A. 2006, AJ, 132, 2333

Strader, J., Brodie, J.P., \& Forbes, D.A. 2004, AJ, 127, 3431

Strom, K.M., \& Strom, S.E. 1978, AJ, 83, 73

Tamura, N., Sharples, R.M., Arimoto, N., Onodera, M.; Ohta, K., \& Yamada, Y. 2006, MNRAS, 373,588

Thomsen, B., \& Baum, W.A. 1987, ApJ, 315, 460

Waters, C.Z., Zepf, S.E., Lauer, T.R., Baltz, E.A., \& Silk, J. 2006, ApJ, 650, 885

Wehner, E.M.H., \& Harris, W.E. 2007, ApJ, 668, L35

Wehner, E.M.H., Harris, W.E., Whitmore, B.C., Rothberg, B., \& Woodley, K.A. 2008, ApJ, 681, 1233 
Whitmore, B.C., Schweizer, F., Kundu, A., \& Miller, B.W. 2002, AJ, 124, 147

Whitmore, B.C., Zhang, Q., Leitherer, C., Fall, S.M., Schweizer, F., \& Miller, B.W. 1999, AJ, 118, 1551

Woodworth, S.C., \& Harris, W.E. 2000, AJ, 119, 2699 (Paper III)

Zepf, S.E., \& Ashman, K.M. 1993, MNRAS, 264, 611 
Table 1. Coma Target Galaxies

\begin{tabular}{lccccr}
\hline \hline Galaxy & $M_{V}^{t}$ & $\begin{array}{c}\text { Velocity } \\
(\mathrm{km} / \mathrm{s})\end{array}$ & $\begin{array}{c}\text { Radius } \\
(\operatorname{arcmin})\end{array}$ & $N_{\text {tot }}(G C)$ & $S_{N}$ \\
& & & & \\
\hline NGC 4874 & -23.43 & 7224 & 0 & $>18700 \pm 2260$ & $>7.9 \pm 1.0$ \\
NGC 4881 & -21.76 & 6740 & 6.5 & $290 \pm 80$ & $0.8 \pm 0.2$ \\
NGC 4889 & -23.67 & 6495 & 7.2 & $11000 \pm 1340$ & $4.7 \pm 0.6$ \\
NGC 4926 & -22.20 & 7887 & 36.5 & $1300 \pm 300$ & $2.0 \pm 0.5$ \\
IC 4051 & -22.06 & 8793 & 17.5 & $6700 \pm 530$ & $12.1 \pm 1.0$ \\
\hline
\end{tabular}

Table 2. Raw Image Data

\begin{tabular}{lcclc}
\hline \hline Galaxy & $V$ Filter & Exposure & $I$ Filter & Exposure \\
& & & & \\
\hline NGC 4874 & $F 606 W$ & $20940 \mathrm{~s}$ & $F 814 W$ & $8720 \mathrm{~s}$ \\
NGC 4881 & $F 555 W$ & $7200 \mathrm{~s}$ & $F 814 W$ & $7200 \mathrm{~s}$ \\
NGC 4889 & $F 606 W$ & $23400 \mathrm{~s}$ & $F 814 W$ & $7800 \mathrm{~s}$ \\
NGC 4926 & $F 606 W$ & $31200 \mathrm{~s}$ & $F 814 W$ & $10400 \mathrm{~s}$ \\
IC 4051 & $F 606 W$ & $20500 \mathrm{~s}$ & $F 814 W$ & $5200 \mathrm{~s}$ \\
\hline
\end{tabular}

Table 3. Completeness Function Parameters

\begin{tabular}{lcccc}
\hline \hline \multicolumn{1}{c}{ Galaxy/ccd } & $\alpha_{V}$ & $m_{0}(V)$ & $\alpha_{I}$ & $m_{0}(I)$ \\
& & & & \\
\hline NGC 4874/PC1 & 3.7 & 27.60 & 4.0 & 25.20 \\
NGC 4874/WF2,3,4 & 3.7 & 27.60 & 3.6 & 25.75 \\
NGC 4881/PC1 & 4.3 & 26.15 & 4.6 & 25.30 \\
NGC 4881/WF2,3,4 & 4.5 & 26.70 & 3.6 & 25.70 \\
NGC 4889/PC1 & 1.5 & 28.00 & 2.3 & 25.75 \\
NGC 4889/WF2,3,4 & 2.3 & 28.10 & 3.5 & 25.90 \\
NGC 4926/PC1 & 3.3 & 28.15 & 4.6 & 25.80 \\
NGC 4926/WF2,3,4 & 1.8 & 28.89 & 2.6 & 26.60 \\
IC 4051/PC1 & 2.6 & 27.70 & 3.5 & 25.40 \\
IC 4051/WF2,3,4 & 2.5 & 28.35 & 3.6 & 26.10 \\
\hline
\end{tabular}


Table 4. GCS Radial Profile Data

\begin{tabular}{cccc}
\hline \hline $\begin{array}{c}\langle r\rangle \\
(\operatorname{arcsec})\end{array}$ & $\mathrm{n}$ & $\begin{array}{c}\text { Area } \\
\left(\operatorname{arcsec}^{2}\right)\end{array}$ & $\begin{array}{c}\sigma \\
\left(\operatorname{arcsec}^{-2}\right)\end{array}$ \\
& & & \\
\hline NGC 4881 & & & \\
2.45 & 4 & 15.8 & $0.253 \pm 0.126$ \\
3.67 & 4 & 35.2 & $0.114 \pm 0.057$ \\
5.51 & 3 & 79.5 & $0.038 \pm 0.022$ \\
8.27 & 6 & 179.1 & $0.034 \pm 0.014$ \\
12.40 & 14 & 402.4 & $0.035 \pm 0.009$ \\
18.60 & 15 & 733.1 & $0.021 \pm 0.005$ \\
27.90 & 24 & 1185.6 & $0.020 \pm 0.004$ \\
41.85 & 49 & 2888.2 & $0.017 \pm 0.002$ \\
62.78 & 96 & 6212.8 & $0.016 \pm 0.002$ \\
94.17 & 81 & 5925.4 & $0.014 \pm 0.002$ \\
141.25 & 2 & 168.1 & $0.012 \pm 0.008$ \\
& & & \\
NGC 4874 & & & \\
4.73 & 12 & 48.5 & $0.248 \pm 0.072$ \\
6.63 & 18 & 94.4 & $0.191 \pm 0.045$ \\
9.28 & 28 & 185.6 & $0.151 \pm 0.029$ \\
12.99 & 58 & 315.3 & $0.184 \pm 0.024$ \\
18.18 & 54 & 497.0 & $0.109 \pm 0.015$ \\
15.45 & 88 & 758.3 & $0.116 \pm 0.012$ \\
35.64 & 192 & 1585.8 & $0.121 \pm 0.009$ \\
49.89 & 305 & 3212.8 & $0.095 \pm 0.005$ \\
69.89 & 383 & 5578.7 & $0.067 \pm 0.004$ \\
97.79 & 262 & 4900.2 & $0.053 \pm 0.003$ \\
131.74 & 15 & 264.4 & $0.057 \pm 0.015$ \\
& & & \\
NGC 4889 & & & \\
4.90 & 61 & 63.2 & $0.966 \pm 0.124$ \\
11.02 & 121 & 141.1 & $0.857 \pm 0.078$ \\
16.53 & 168 & 318.1 & $0.544 \pm 0.041$ \\
& & & $0.292 \pm 0.022$
\end{tabular}


Table 4-Continued

\begin{tabular}{|c|c|c|c|}
\hline $\begin{array}{c}\langle r\rangle \\
(\operatorname{arcsec})\end{array}$ & $\mathrm{n}$ & $\begin{array}{c}\text { Area } \\
\left(\operatorname{arcsec}^{2}\right)\end{array}$ & $\begin{array}{c}\sigma \\
\left(\operatorname{arcsec}^{-2}\right)\end{array}$ \\
\hline 24.80 & 145 & 738.7 & $0.196 \pm 0.016$ \\
\hline 37.20 & 348 & 2103.8 & $0.165 \pm 0.009$ \\
\hline 55.80 & 492 & 4572.9 & $0.108 \pm 0.005$ \\
\hline 83.70 & 583 & 7490.5 & $0.078 \pm 0.003$ \\
\hline 125.56 & 59 & 1012.8 & $0.058 \pm 0.008$ \\
\hline \multicolumn{4}{|l|}{ NGC 4926} \\
\hline 4.90 & 29 & 62.8 & $0.459 \pm 0.085$ \\
\hline 7.35 & 35 & 141.4 & $0.248 \pm 0.042$ \\
\hline 11.02 & 57 & 318.1 & $0.179 \pm 0.024$ \\
\hline 16.53 & 59 & 571.1 & $0.103 \pm 0.013$ \\
\hline 24.80 & 53 & 837.8 & $0.063 \pm 0.009$ \\
\hline 37.20 & 668 & 2150.6 & $0.031 \pm 0.004$ \\
\hline 55.80 & 81 & 5050.4 & $0.016 \pm 0.002$ \\
\hline 83.70 & 107 & 7643.4 & $0.014 \pm 0.002$ \\
\hline 125.56 & 8 & 1012.8 & $0.008 \pm 0.003$ \\
\hline \multicolumn{4}{|l|}{ IC 4051} \\
\hline 3.67 & 101 & 35.2 & $2.870 \pm 0.286$ \\
\hline 5.51 & 134 & 79.5 & $1.685 \pm 0.146$ \\
\hline 8.27 & 215 & 179.1 & $1.200 \pm 0.082$ \\
\hline 12.40 & 313 & 402.4 & $0.778 \pm 0.044$ \\
\hline 18.60 & 269 & 620.9 & $0.433 \pm 0.026$ \\
\hline 27.90 & 245 & 1099.0 & $0.223 \pm 0.014$ \\
\hline 41.85 & 284 & 2820.8 & $0.101 \pm 0.006$ \\
\hline 62.78 & 325 & 6063.7 & $0.054 \pm 0.003$ \\
\hline 94.17 & 277 & 6260.1 & $0.044 \pm 0.003$ \\
\hline 141.25 & 8 & 246.8 & $0.032 \pm 0.012$ \\
\hline
\end{tabular}


Table 5. GCS Luminosity Function Data

\begin{tabular}{|c|c|c|c|c|c|c|}
\hline$V(\min )$ & $V(\max )$ & N4874 & N4889 & N4926 & I4051 & Bkgd \\
\hline 23.00 & 23.25 & 5 & 1 & 0 & 3 & 0 \\
\hline 23.25 & 23.50 & 14 & 0 & 0 & 4 & 2 \\
\hline 23.50 & 23.75 & 16 & 4 & 0 & 12 & 2 \\
\hline 23.75 & 24.00 & 27 & 6 & 1 & 12 & 0 \\
\hline 24.00 & 24.25 & 58 & 7 & 2 & 17 & 1 \\
\hline 24.25 & 24.50 & 77 & 19 & 6 & 29 & 4 \\
\hline 24.50 & 24.75 & 98 & 39 & 4 & 38 & 5 \\
\hline 24.75 & 25.00 & 118 & 65 & 7 & 59 & 5 \\
\hline 25.00 & 25.25 & 139 & 87 & 9 & 87 & 6 \\
\hline 25.25 & 25.50 & 180 & 119 & 24 & 126 & 13 \\
\hline 25.50 & 25.75 & 216 & 141 & 29 & 133 & 15 \\
\hline 25.75 & 26.00 & 252 & 252 & 36 & 156 & 34 \\
\hline 26.00 & 26.25 & 294 & 262 & 56 & 227 & 24 \\
\hline 26.25 & 26.50 & 366 & 315 & 62 & 268 & 61 \\
\hline 26.50 & 26.75 & 401 & 404 & 93 & 305 & 55 \\
\hline 26.75 & 27.00 & 493 & 475 & 95 & 372 & 89 \\
\hline 27.00 & 27.25 & 552 & 588 & 121 & 391 & 128 \\
\hline 27.25 & 27.50 & 544 & 661 & 190 & 464 & 157 \\
\hline 27.50 & 27.75 & 371 & 713 & 228 & 411 & 188 \\
\hline 27.75 & 28.00 & 206 & 743 & 254 & 398 & 201 \\
\hline 28.00 & 28.25 & - & 649 & 256 & 385 & 282 \\
\hline 28.25 & 28.50 & - & - & 317 & 399 & 344 \\
\hline Area & & 4.80 & 4.44 & 2.60 & 3.34 & 3.99 \\
\hline
\end{tabular}


Table 6. Gaussian Model Fits to $(V-I)$ Color Distributions

\begin{tabular}{ccccc}
\hline \hline Galaxy & Mean $\mu$ & Dispersion $\sigma$ & Proportion & Comment \\
& & & & \\
\hline \multirow{2}{*}{ NGC 4874 } & $1.106 \pm 0.006$ & $0.134 \pm 0.004$ & 1.00 & Unimodal \\
& $1.001 \pm 0.042$ & $0.123 \pm 0.024$ & 0.30 & Bimodal \\
& $1.147 \pm 0.018$ & $0.116 \pm 0.012$ & 0.70 & $"$ \\
NGC 4889 & $1.096 \pm 0.007$ & $0.132 \pm 0.005$ & 1.00 & Unimodal \\
& $0.978 \pm 0.016$ & $0.080 \pm 0.010$ & 0.30 & Bimodal \\
& $1.147 \pm 0.011$ & $0.117 \pm 0.009$ & 0.70 & $"$ \\
IC 4051 & $1.093 \pm 0.009$ & $0.157 \pm 0.006$ & 1.00 & Unimodal \\
& $0.958 \pm 0.031$ & $0.123 \pm 0.020$ & 0.30 & Bimodal \\
& $1.150 \pm 0.015$ & $0.132 \pm 0.013$ & 0.70 & $"$ \\
NGC 4881 & $0.788 \pm 0.016$ & $0.093 \pm 0.012$ & 1.00 & Unimodal \\
NGC 4926 & $1.191 \pm 0.016$ & $0.189 \pm 0.013$ & 1.00 & Unimodal \\
\hline
\end{tabular}




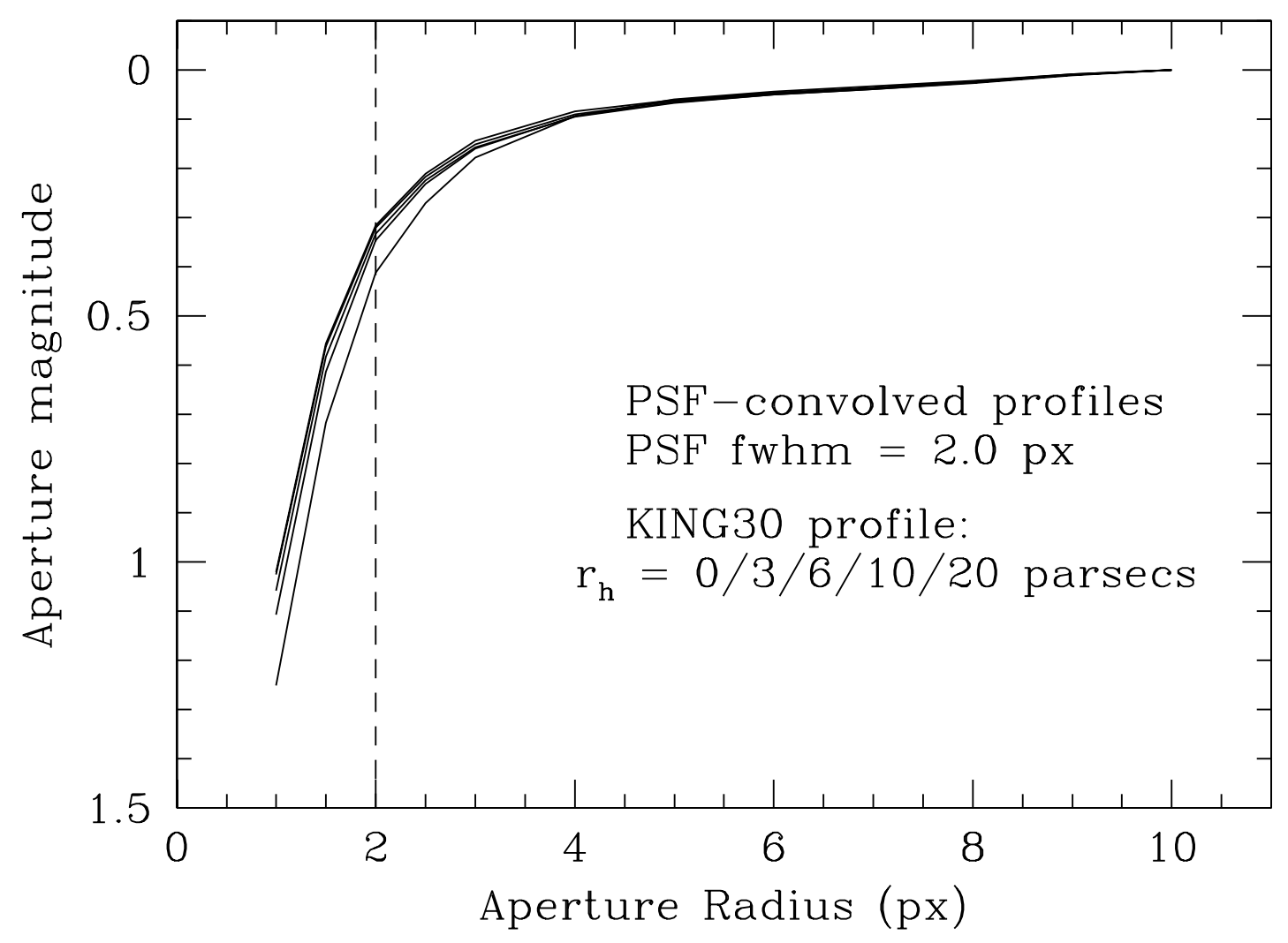

Fig. 1. - Aperture growth curves for various synthetic globular clusters. Each curve shows the results for a KING30 model cluster with a particular half-light radius $r_{h}$, placed at the 100-Mpc distance of Coma and then convolved with the WFPC2 point spread function. The magnitude within aperture radius $r(a p)$ is shown for five model clusters with $r_{h}=0,3,6,10$, and 20 parsecs, a range which generously brackets real globular clusters. The curves are plotted to osculate at $r=10$ px; the top curve is for $r_{h}=0$ (that is, a starlike object), while the bottom curve is for $r_{h}=20$ pc, which would be a very extended object resembling a UCD; see text. The curves for $r_{h}=0$ and 3 pc are indistinguishable. For typical globular cluster radii $r_{h}<6 \mathrm{pc}$, the PSF-convolved profiles can be treated as stars at this distance, and even for UCD-type objects the profiles are starlike for $r>5 \mathrm{px}$. 


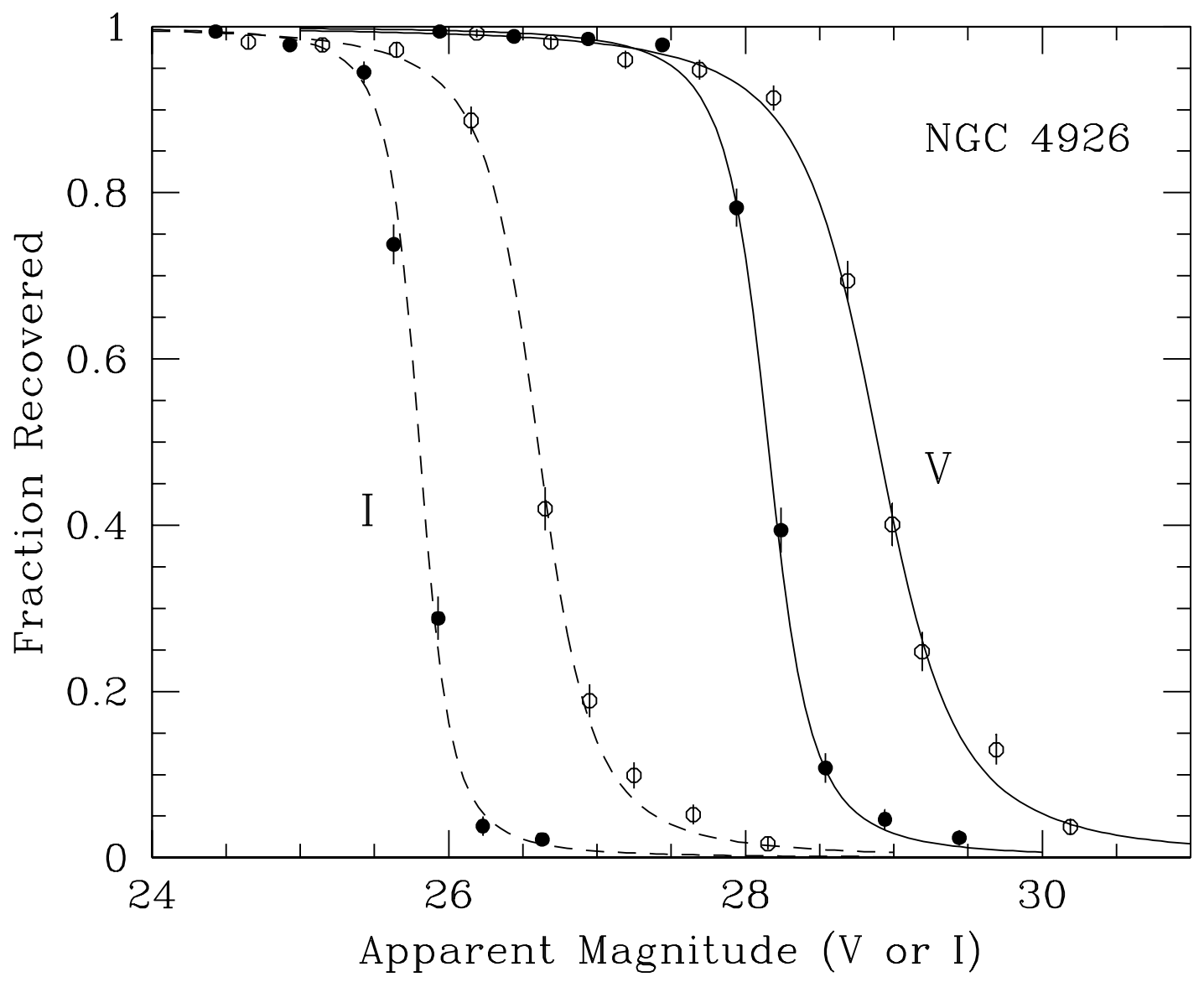

Fig. 2.- Completeness of detection of the photometry for the NGC 4926 field. The smooth curves are the interpolation functions given in the text: the solid lines represent the mean for the $V$ frames while the dashed lines represent the $I$ frames. In each pair the left-hand curve (solid dots) shows the curve for the PC1 CCD chip, while the right-hand curve (open circles) shows the WF CCD chips. 

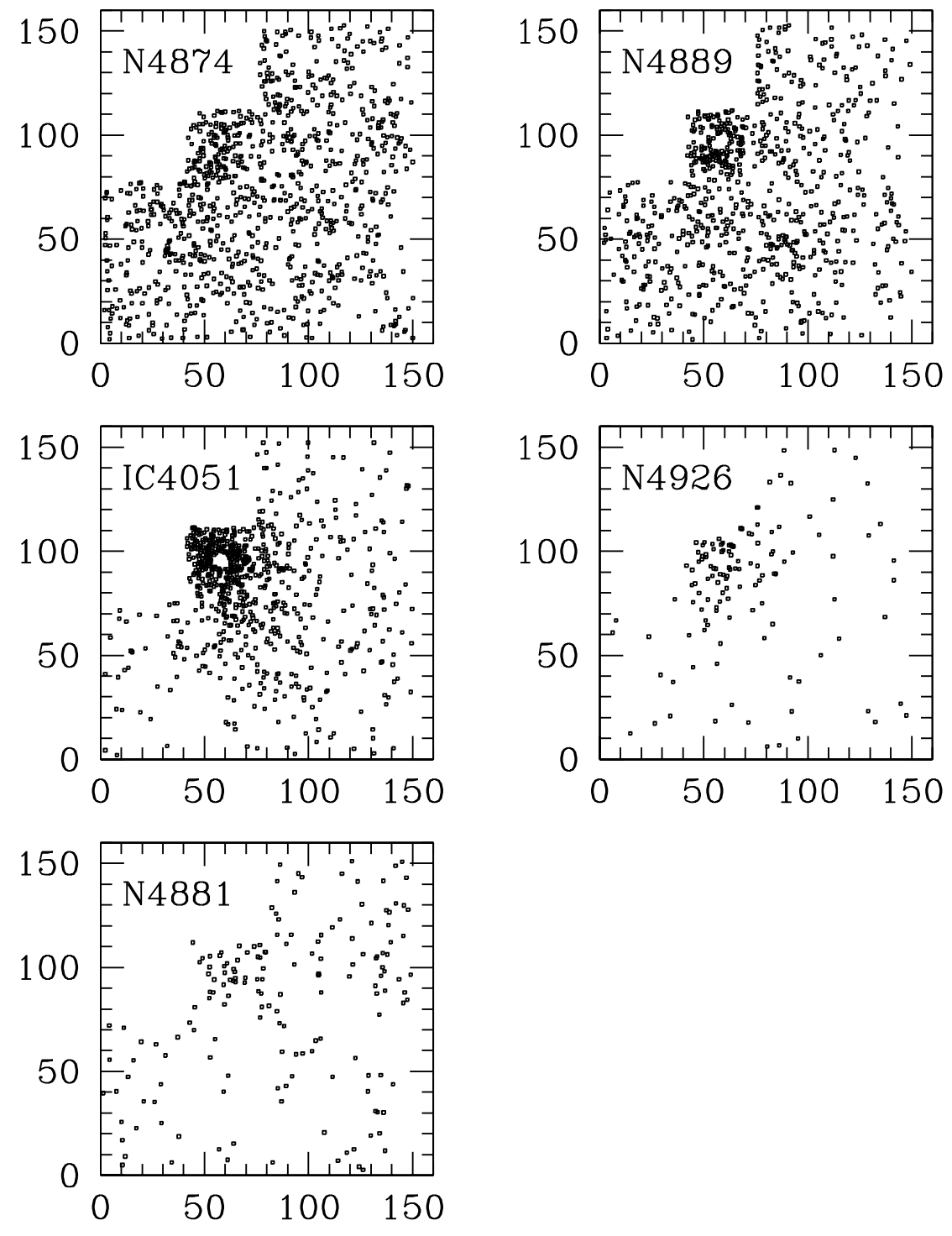

Fig. 3.- Distribution of detected starlike objects around each of the five target galaxies in the WFPC2 field. In each case the objects brighter than $V=26.0$ are shown, with no additional selections by color. The scale shown is in arcseconds. The orientation is such that the PC1 chip is at upper left, WF2 at lower left, WF3 at lower right, and WF4 at upper right. In every case the center of the galaxy is nearly at the center of the PC1 chip. 

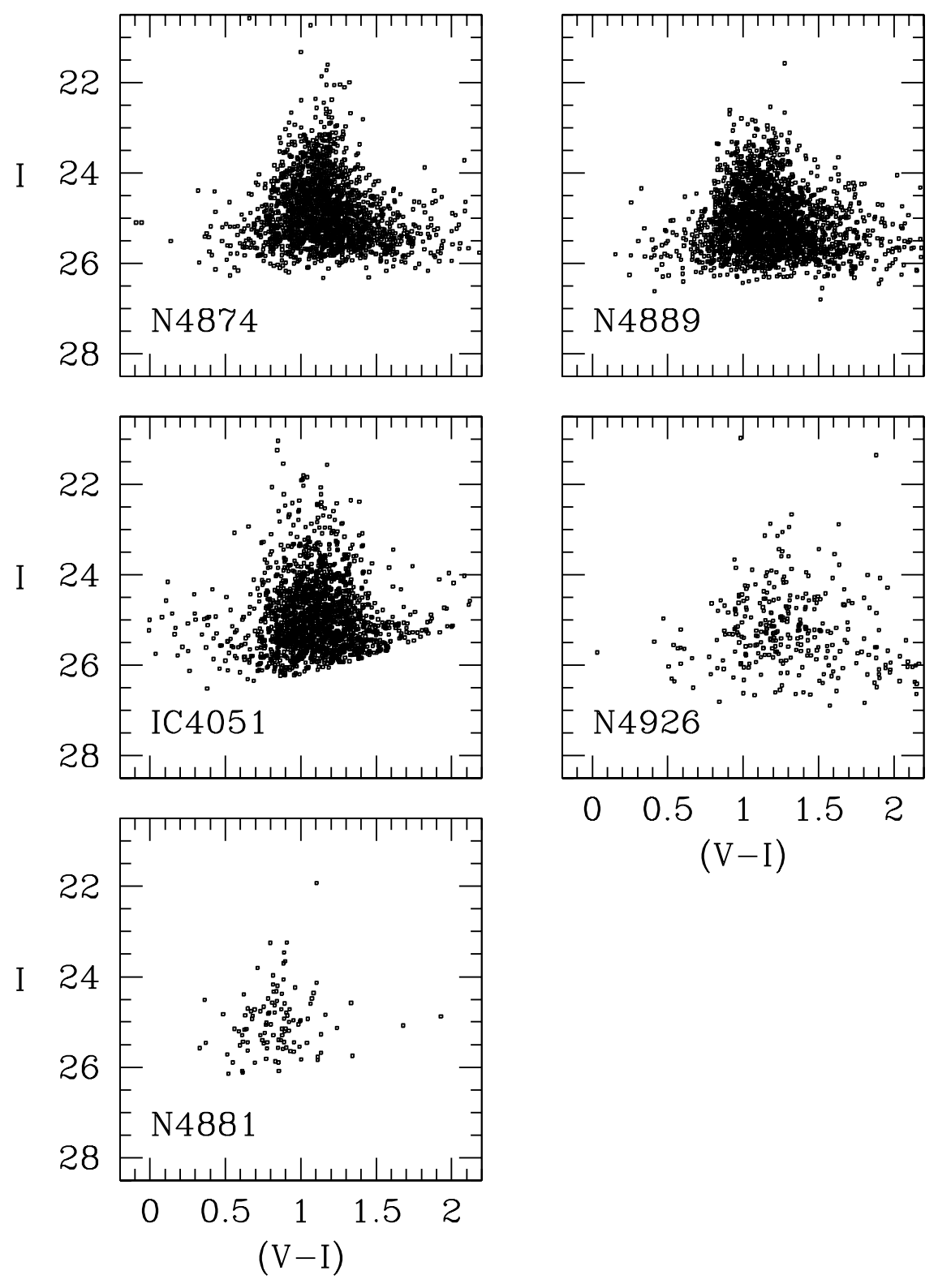

Fig. 4.- Color-magnitude plots for the measured starlike objects around the five galaxies. All such objects on each WFPC2 field are shown. 

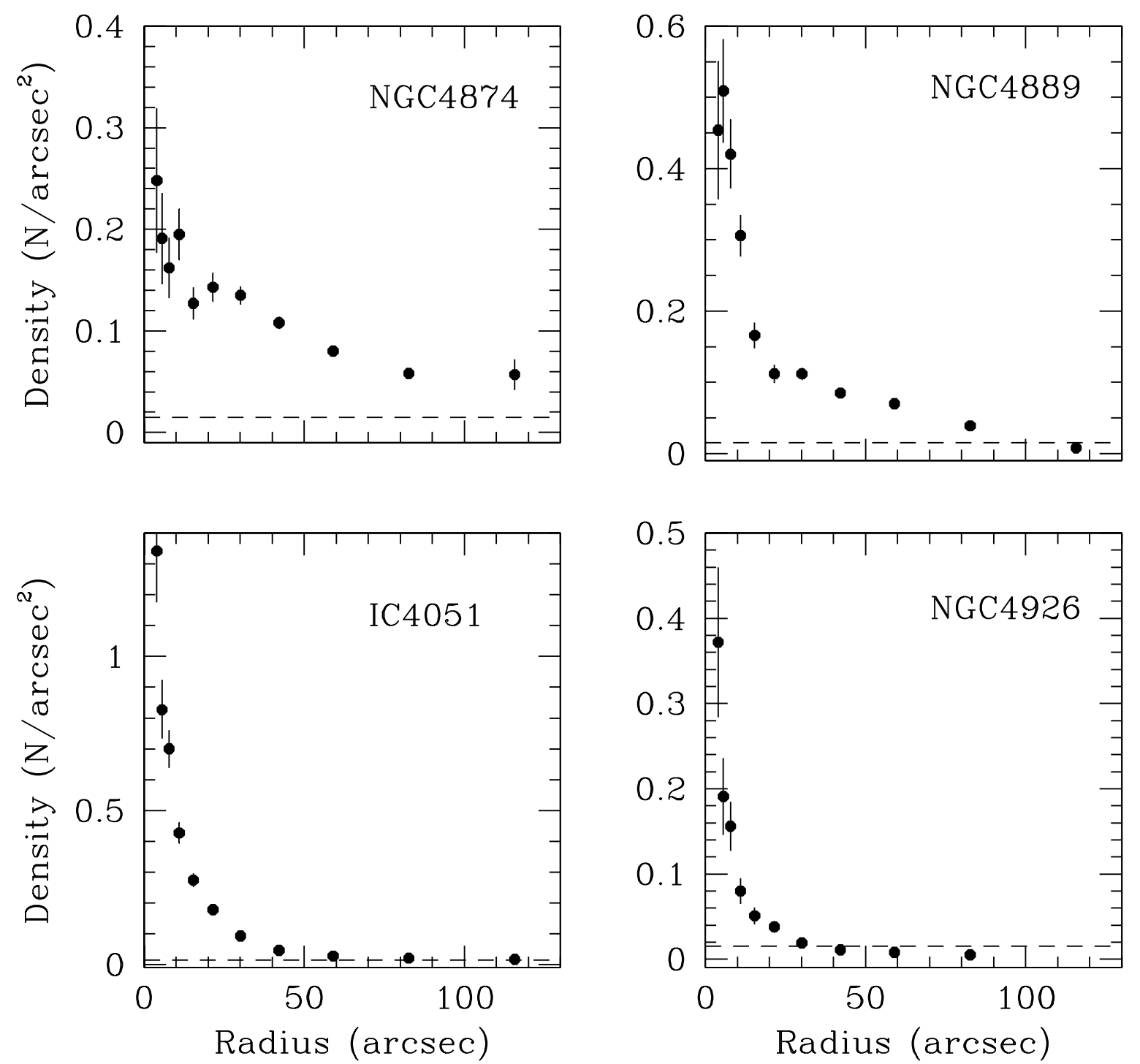

Fig. 5.- Radial distributions for the measured starlike objects around the four galaxies labelled. Number of measured objects per $\operatorname{arcsec}^{-2}$ is plotted against radius from galaxy center; the linear scale is $50 \mathrm{kpc}$ per $100^{\prime \prime}$. Here the sample is restricted to objects in the range $22.0<I<25.5$ and $0.5<(V-I)<1.8$, which includes the brighter part of the globular cluster systems. The approximate "background" level of field contamination is shown by the dashed lines. Notice the different vertical scales on each graph; for example, although NGC 4874 has by far the most spatially extended GCS of all these galaxies, continuing well beyond the border of our WFPC2 field, it does not have the GCS with the highest central density. 

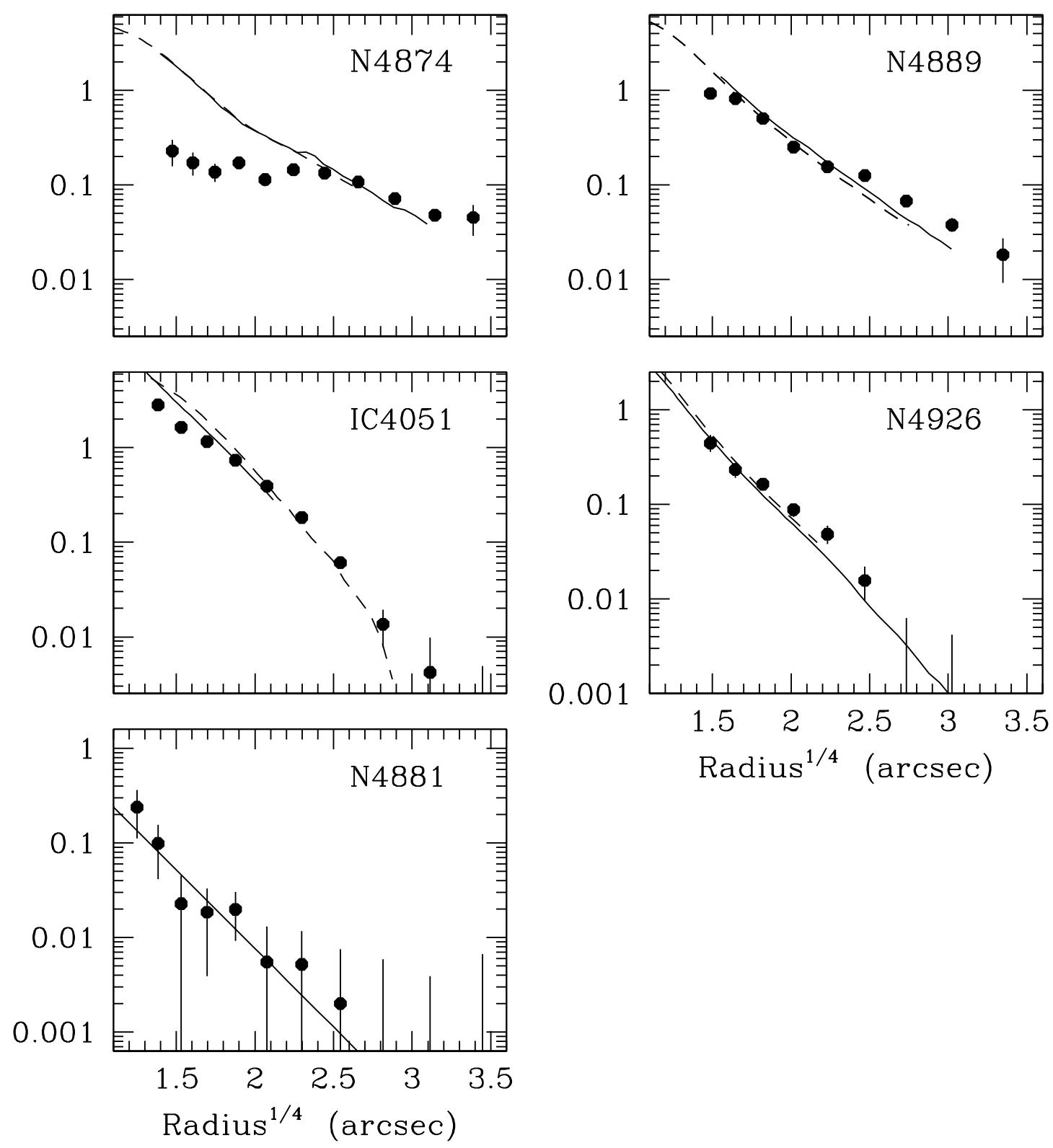

Fig. 6. - Radial distribution of the globular cluster systems around our five galaxies. The ordinate of each graph is the number density, $\sigma_{c l}$ of globular clusters per $\operatorname{arcsec}^{2}$. The $r^{1 / 4}$ values are for radius in arcseconds. In each graph the lines give the radial falloff of the surface light intensity of each galaxy; see text for sources. 

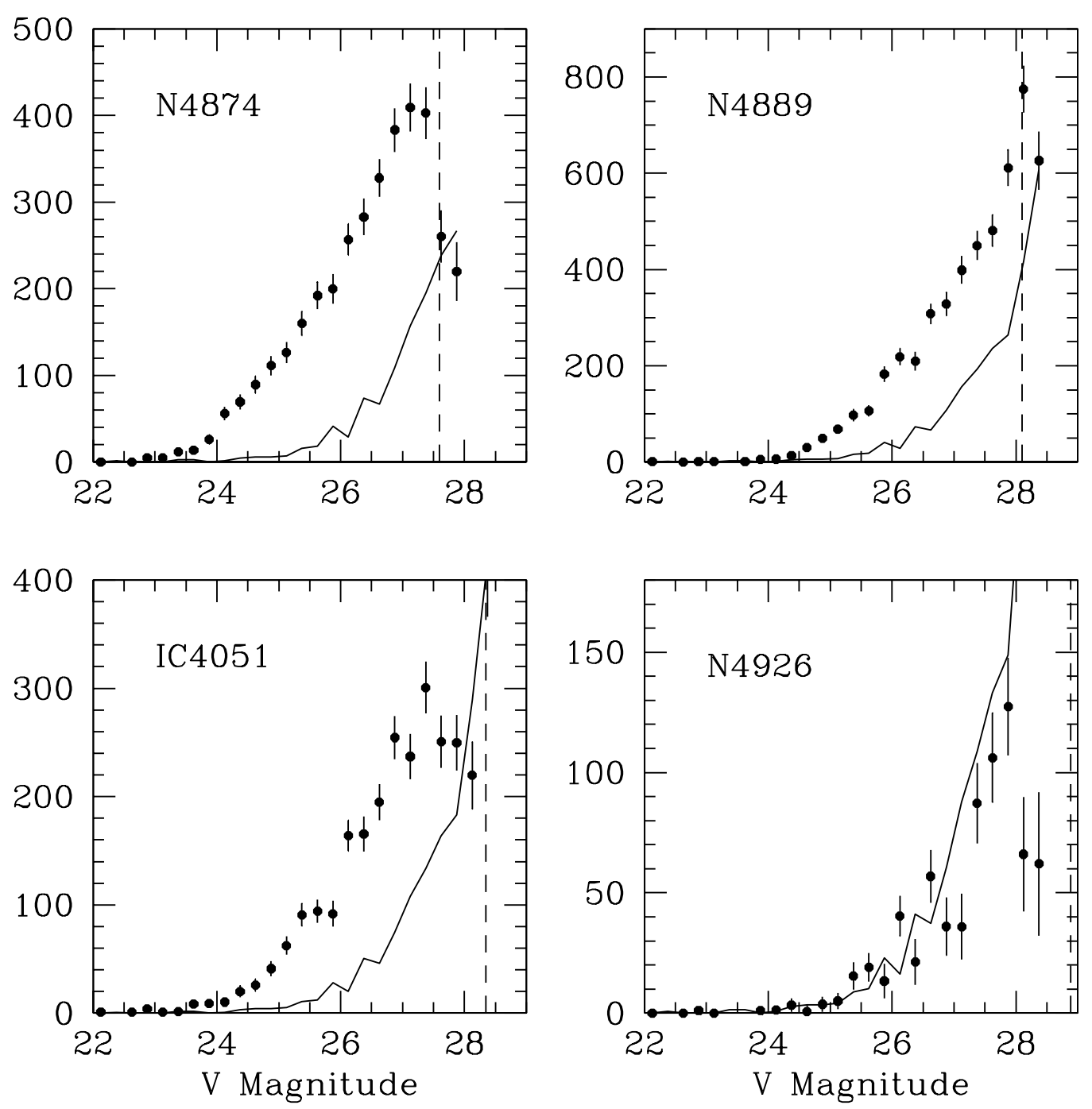

Fig. 7.- Luminosity functions for the globular clusters shown individually for the four Coma galaxies with our deepest photometry. The LFs are after background subtraction and completenesscorrected. For comparison, the adopted background LFs are shown as the broken line. Number of clusters per 0.25 -mag bin is plotted against $V$. In each panel, the vertical dashed line indicates the $50 \%$ completeness level of the photometry in the WF2,3,4 frames. 


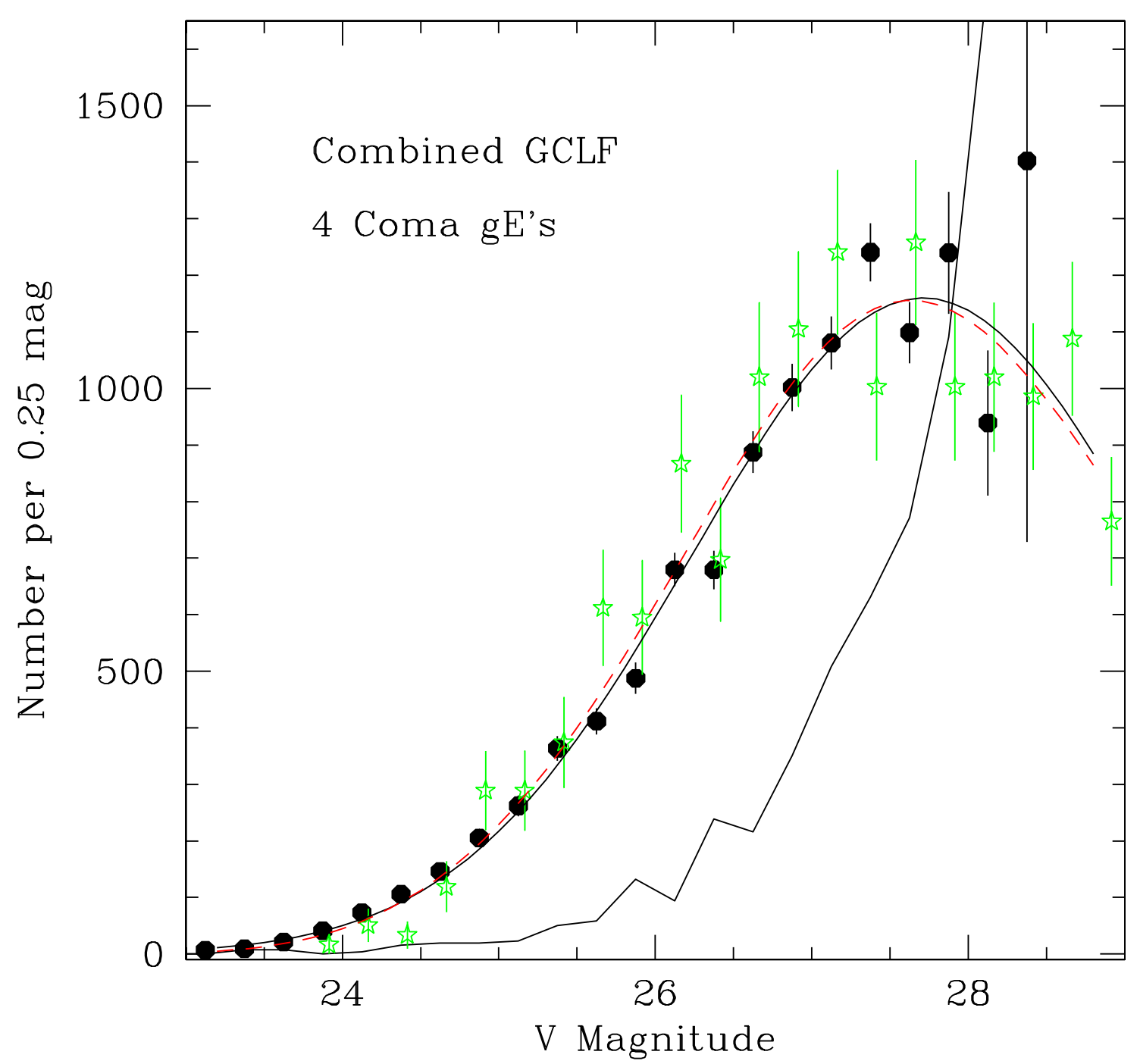

Fig. 8.- Composite luminosity function for the globular clusters in four Coma ellipticals, shown in solid dots with error bars. The composite LF is completeness-corrected and background-subtracted according to the prescription described in the text. The broken line at bottom is the background LF by itself, for comparison. The GCLF for the Virgo giant M87 is shown for comparison in open starred symbols, shifted by $\Delta V=4.04 \mathrm{mag}$ and normalized to the same total GC population brighter than the turnover point (see text). The best-fitting Gaussian function, with turnover level $V^{t o}=27.71$ and dispersion $\sigma_{V}=1.48$, is shown as the solid line. The dashed line gives the best-fit evolved Schechter function model, which has parameters $\left(\delta-V_{c}\right)=3.2$ and a "cutoff" magnitude $V_{c}=24.4$. 

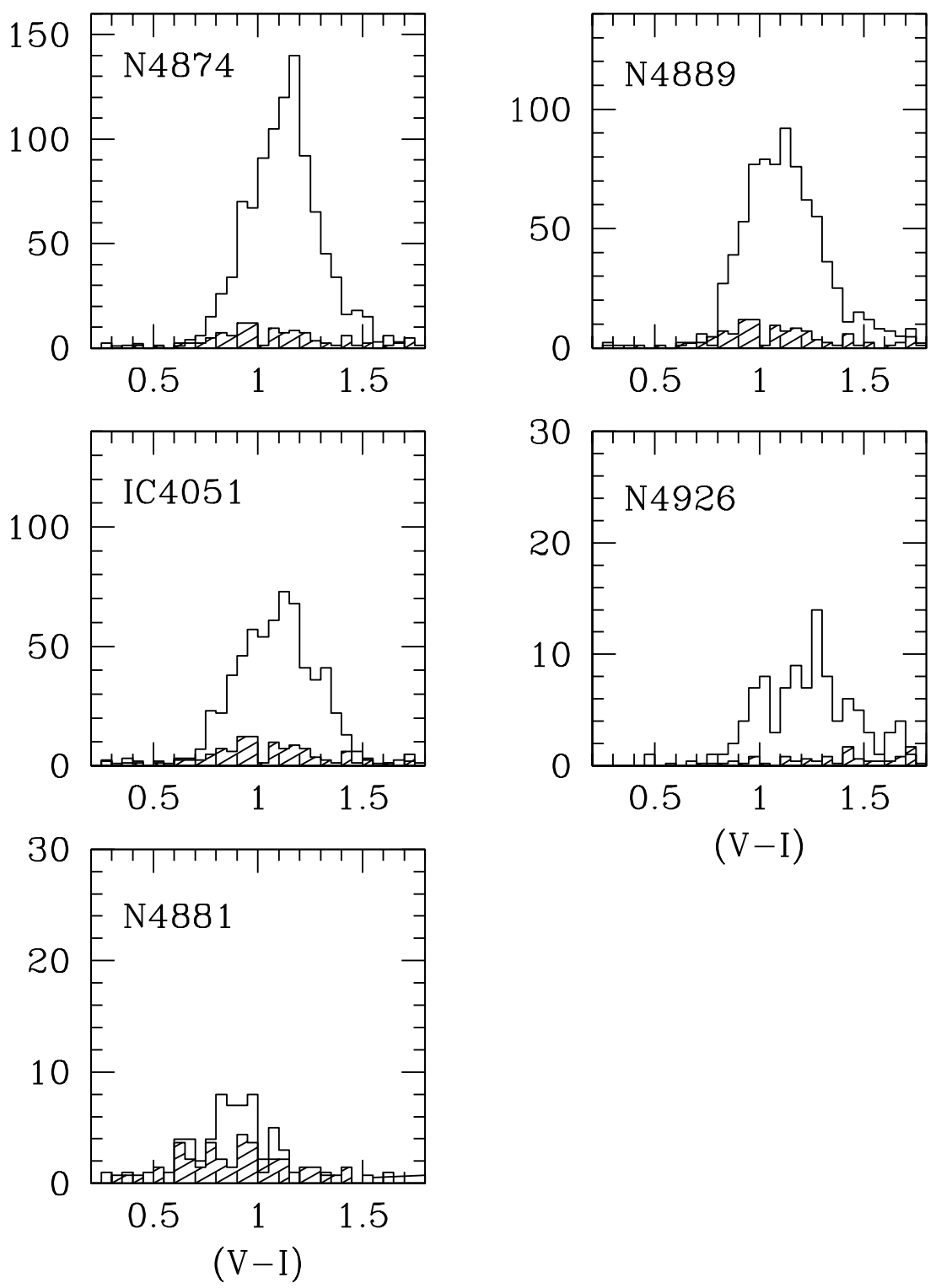

Fig. 9.- Color histograms in $(V-I)$ for the starlike sources around the five galaxies. In each case the "background" population at large radius is shown as the hatched histogram, while the population of objects closer to the galaxy is the open histogram (not background-subtracted). Both histograms are normalized to the same area on the sky. For the first three objects (NGC 4874, NGC 4889, IC 4051) the background color distribution is the mean of the far-field distributions from IC 4051 and NGC 4926, as described in the text. For the last two objects (NGC 4926, NGC 4881) the background is a locally defined one (see text). 

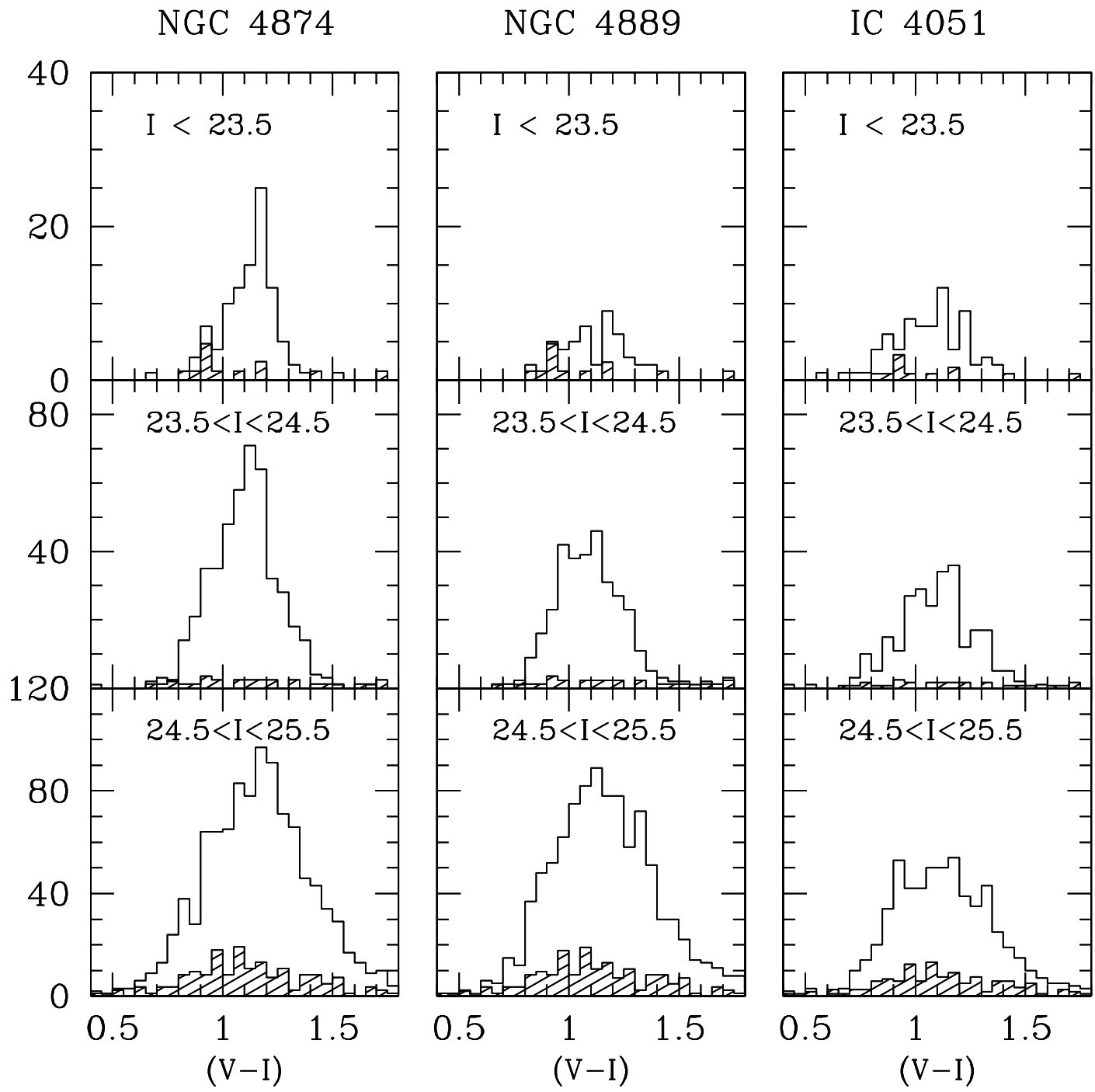

Fig. 10.- Color histograms for the globular clusters around the three most populous systems in our study, now subdivided by magnitude range. The $(V-I)$ distribution of stars in the global background field is shown in the filled histograms. These should be subtracted from the open histograms to give the residual GC population distributions. 

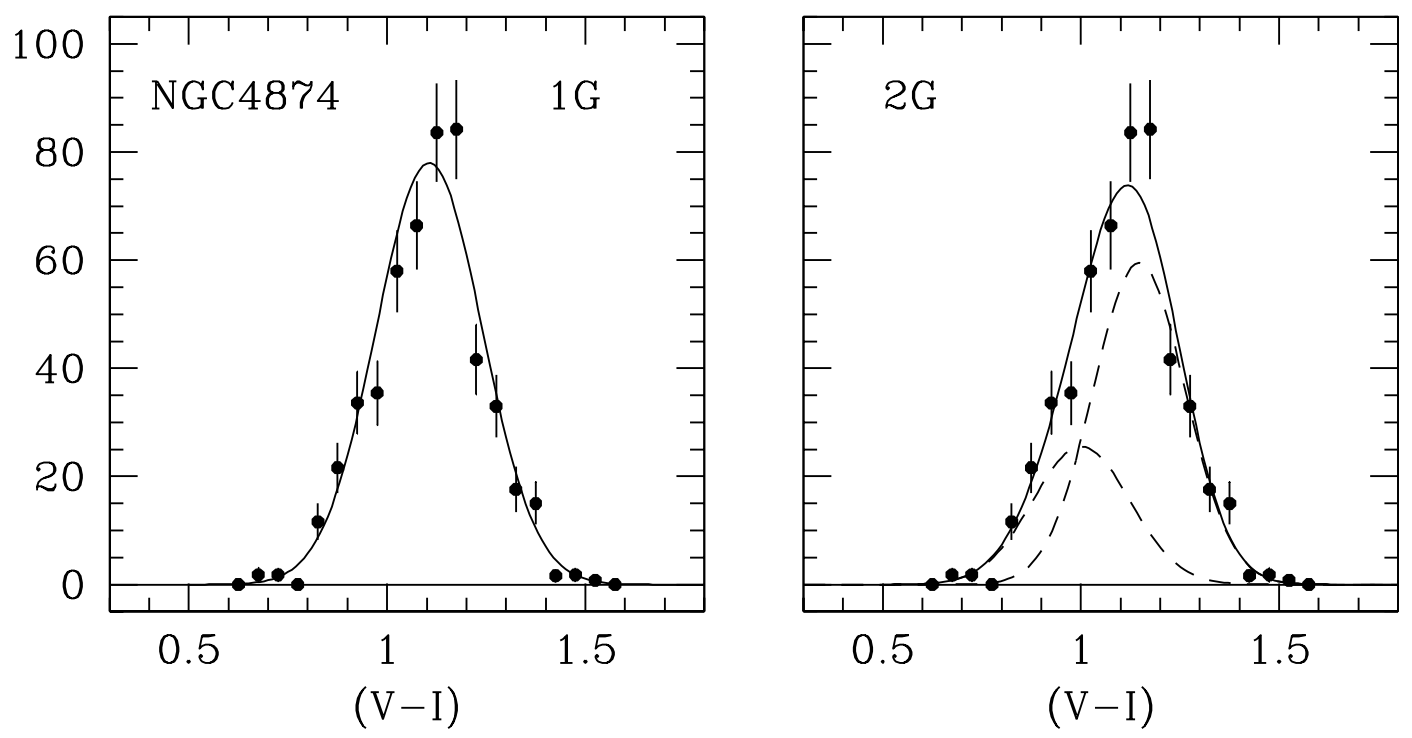

Fig. 11. - Color histogram for the bright globular clusters around NGC $4874(I<24.5)$ for which field contamination and photometric errors are minimal. The left panel shows a best-fit single Gaussian function to the color histogram, while the right panel shows a possible two-Gaussian solution to the same data, with the parameters as listed in Table 6 . 

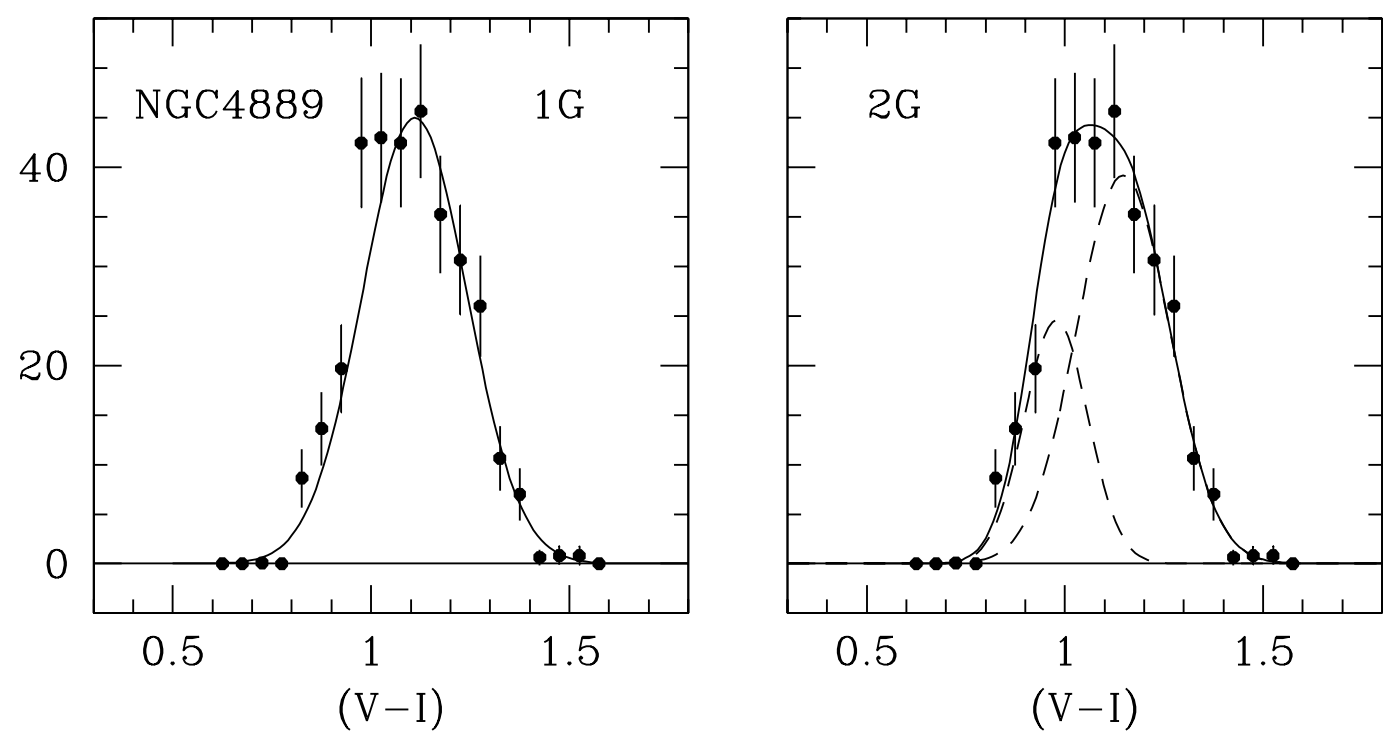

Fig. 12. - Same as the previous figure, for the bright clusters around NGC 4889. In this case the two-Gaussian model provides a significantly better fit. 

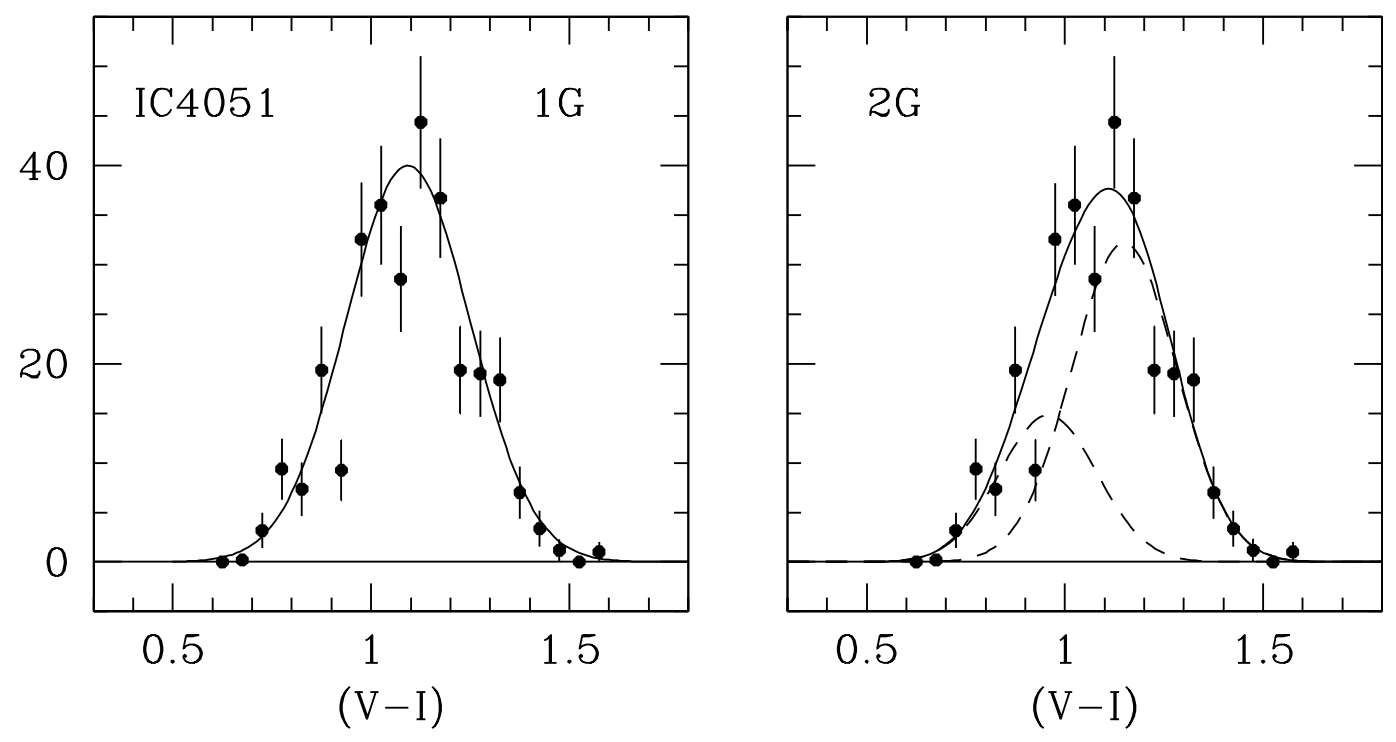

Fig. 13.- Same as the previous figure, for the bright clusters around IC 4051. 

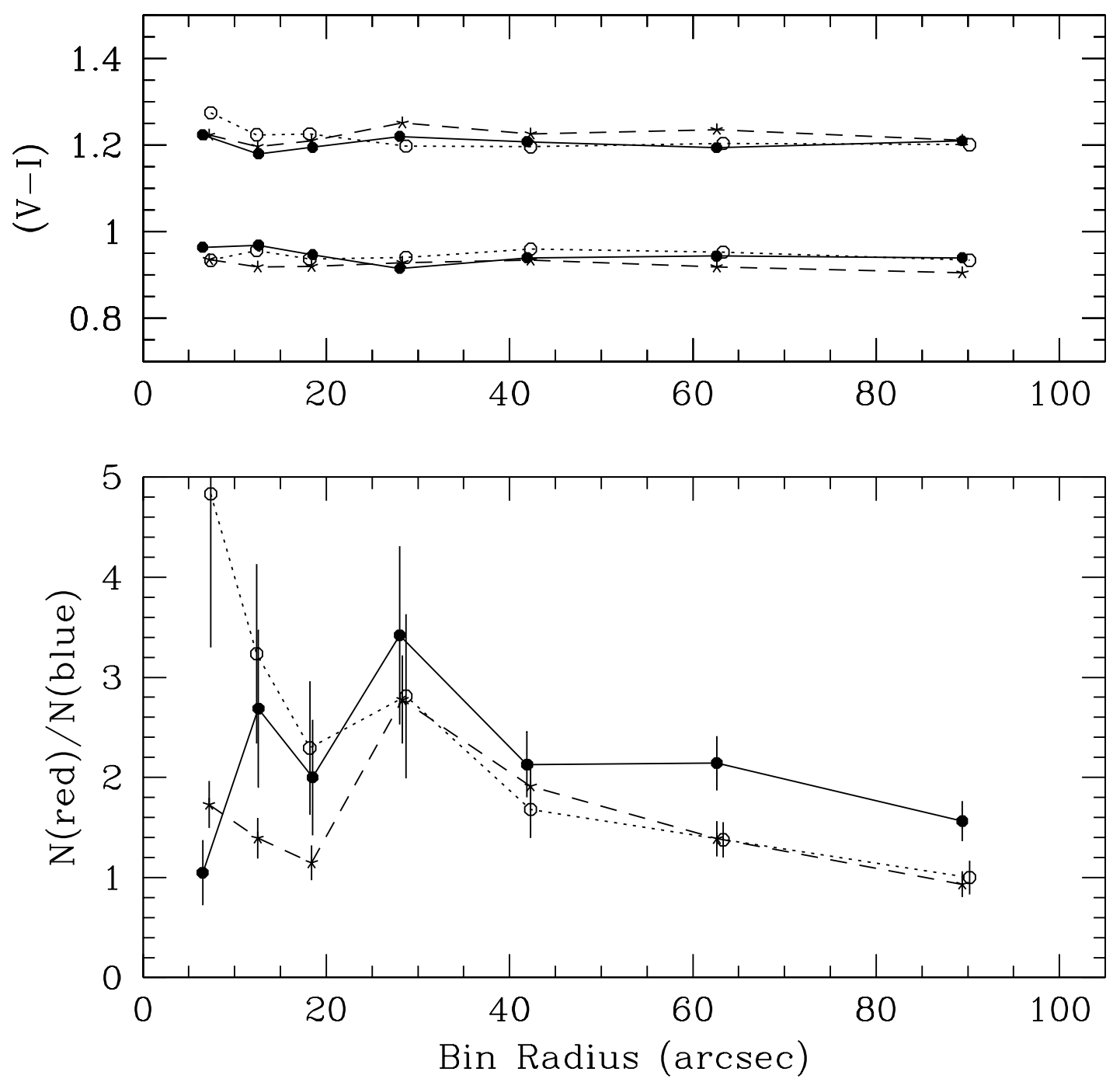

Fig. 14.- Upper panel: Mean color of the blue GCs (lower set of three lines) and the red GCs (upper set of three lines) as a function of radius from galaxy center. Data for NGC 4874 are the solid dots and lines; NGC 4889 the squares and dotted lines; and IC 4051 the stars and dashed lines. No color gradient within either the blue or red populations is detectable. Lower panel: Ratio of number of red GCs to number of blue GCs, plotted as a function of radius. Solid dots are for NGC 4874, open circles for NGC 4889, and stars for IC 4051. A slight population gradient exists for each galaxy. 

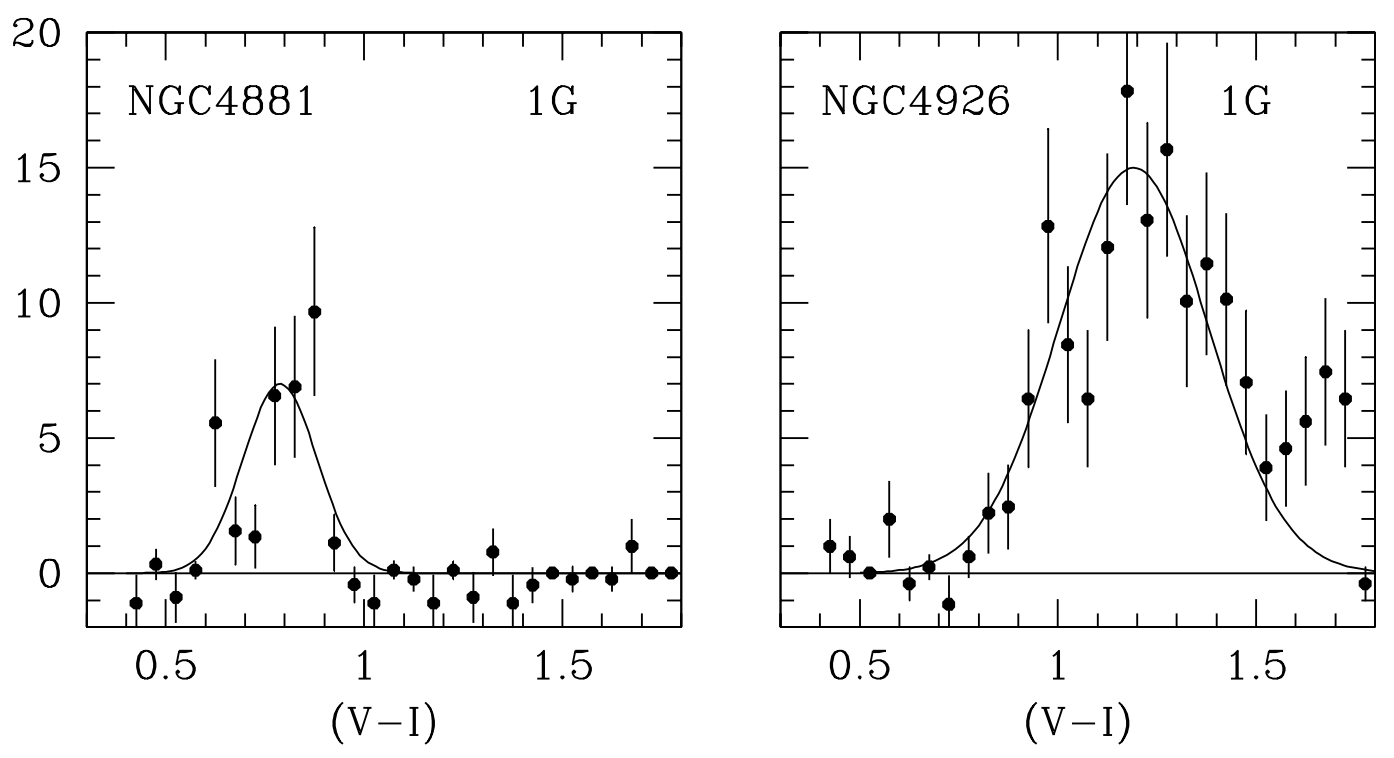

Fig. 15.- Color histograms for the GCs around NGC 4881 and 4926, as described in the text. Single-Gaussian fits are shown for each. 